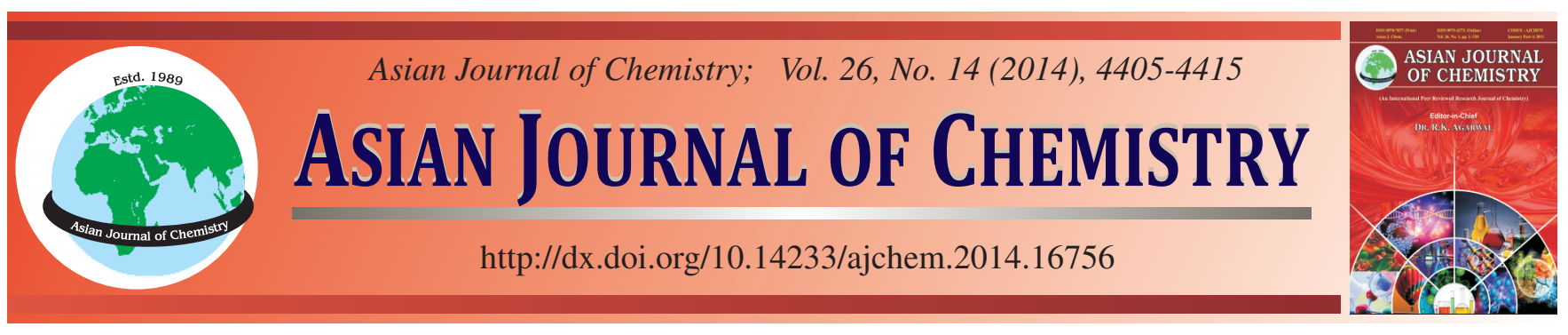

\title{
Synthesis of Some Biologically Active Pyrazolylphthalazine Derivatives and Acyclo- $C$-nucleosides of 6-(2,4,6-trimethylphenyl)-1,2,4-triazolo[3,4-a]phthalazine
}

Ibrahim E. El-Shamy ${ }^{1, *}$, A.M. Abdel-Mohsen ${ }^{2,4}$, Moustafa M.G. Fouda ${ }^{3}$, Salem S. Al-Deyab ${ }^{3}$ and Maher A. El-Hashash ${ }^{5}$

${ }^{1}$ Chemistry Department, Faculty of Science, Fayoum University, Fayoum, Egypt

${ }^{2}$ Central European Institute of Technology, Brno University of Technology, Brno, Czech Republic

${ }^{3}$ Petrochemical Research Chair, Chemistry Department, College of Science, King Saud University, P.O. Box 2455, Riyadh 11451, Saudi Arabia

${ }^{4}$ Textile Research Division, National Research Center, Dokki, Giza, P.O. 12622, Giza 12522, Egypt

${ }^{5}$ Chemistry Department, Faculty of Science, Ain Shams University, Cairo, Egypt

*Corresponding author: E-mail: iei00@ fayoum.edu.eg

1-Chloro-4-(2,4,6-trimethylphenyl)phthalazine (2) was used as a precursor for preparation of some novel pyrazolylphthalazine derivatives 6-13 and 15-19. Moreover, the acyclonucleosides 20-23 a-e were prepared by the reaction of hydrazinophthalazine derivative 3 with different aldoses. All new phthalazine derivatives were characterized using ${ }^{1} \mathrm{H}$ NMR, ${ }^{13} \mathrm{C}$ NMR, FTIR, mass spectrum and elemental analysis. The newly synthesized compounds showed highly activity against different species of bacteria and fungi, in addition to an excellent antiinflammatory property.

Keywords: C-nucleosides, Phthalazine derivatives, Biological activity.

\section{INTRODUCTION}

The synthesis of new series of heterocyclic molecules and the evaluation of their biological and antiinflammatory activities is the major objective of this research work. Heterocyclic molecules that contain nitrogen have received great attention as described by several published articles on their applications in diverse areas, particularly as drugs ${ }^{1,2}$. Phthalazines are examples of heterocycles, that are containing nitrogen, have a great biological interest ${ }^{3-5}$. They form the structural outline for numerous biologically active compounds. Therefore, they are considered a significant key elements in organic synthesis. Numerous reports in the literature have focused on the pharmacology of phthalazine derivatives. These reports have ensued in a great number of contributions in different areas of interest ${ }^{6-11}$. Phthalazine derivatives were described to possess anticonvulsant $^{12}$, cardiotonic ${ }^{13}$, antimicrobial ${ }^{14}$, antitumor ${ }^{15-18}$, antihypertensive ${ }^{19,20}$, antithrombotic ${ }^{21}$, antidiabetic ${ }^{22,23}$, antitrypanosomal $^{24}$, antiinflammatory ${ }^{25-31}$ and vasorelaxant activities ${ }^{20,32}$. Moreover, phthalazines have recently been described to inhibit serotonin reuptake and are considered as antidepression agents $^{33}$. Thus, a number of methods have been described for the synthesis of phthalazine derivatives ${ }^{12,34-40}$. However, the development of new synthetic methods for heterocycles molecules containing phthalazine ring is still considered as a scientific challenge. On the other hand, C-nucleosides were revealed to exhibit noticeable and versatile biological activities ${ }^{41,42}$ and many of their derivatives have been synthesized recently as potential antimicrobial ${ }^{43}$ and antiviral agents ${ }^{44}$. Thus, many reports ${ }^{45-50}$ have recently performed in dealing with this class of nucleosides. In view of the aforementioned facts, we aim to incorporate a phthalazine moiety with other heterocyclic ring system to attain new functions in challenge to improve the antimicrobial activity of compounds containing the phthalazine ring system.

\begin{tabular}{l}
\hline EXPERIMENTAL \\
\hline Melting points were determined on a MEL-TEMP II melting \\
point apparatus in open glass capillaries and are uncorrected. \\
The infrared spectra were recorded for potassium bromide \\
discs on a Pye-Unicam SP1025 spectrophotometer. NMR \\
spectra were carried out at ambient temperature $\left(-25^{\circ} \mathrm{C}\right)$ with \\
a Brucker AC-250 spectrometer or with a Varian Gemini 200 \\
spectrometer at $250 \mathrm{MHz}$ using tetramethylsilane (TMS) as \\
an internal standard. Mass spectra (MS) were performed on a \\
Hewlett-Packard 5995 gas chromatography-mass spectrometer \\
system or on a Shimadzu GCMS-QP 1000 EX mass spectro- \\
meter. Homogeneity of the products and follow up of the reactions
\end{tabular}


were checked by ascending thin-layer chromatography TLC on plates pre-coated with silica gel G (E. Merck; layer thickness $0.25 \mathrm{~mm}$ ), used without pretreatment.

General procedure for synthesis of compounds (4-6): As described from previous work ${ }^{63}$ with some modifications; briefly: active methylene compounds (viz malononitrile, ethyl cyanoacetate, ethyl acetoacetate and acetylacetone, $0.01 \mathrm{~mol}$ ) were added to an ethanolic sodium ethoxide solution $(0.56 \mathrm{~g}$ of sodium in $50 \mathrm{~mL}$ ethanol) and left for stirring for $2 \mathrm{~h}$. Compound $2(0.01 \mathrm{mmol})$ was also added to an ethanolic sodium ethoxide solution $(0.56 \mathrm{~g}$ of sodium in $50 \mathrm{~mL}$ ethanol) and stirred for $2 \mathrm{~h}$. The reaction mixture was heated under reflux for $5 \mathrm{~h}$. The ethanol was removed under reduced pressure and the residue was poured into cold water $(100 \mathrm{~mL})$ and extracted with ether. The extracted solvent was dried over anhydrous sodium sulphate and removed under reduced pressure to give the compounds 4-6, respectively.

2-[4-(2,4,6-Trimethylphenyl)-phthalazin-1-yl]malononitrile (4): Yield: $71 \%$; m.p. $173-174{ }^{\circ} \mathrm{C} ;{ }^{1} \mathrm{H}$ NMR $\left(\mathrm{DMSO}-d_{6}\right) \delta: 2.37\left(\mathrm{~s}, 3 \mathrm{H}, \mathrm{CH}_{3}\right), 2.48\left(\mathrm{~s}, 6 \mathrm{H}, 2 \mathrm{CH}_{3}\right), 4.57(\mathrm{~s}$, $1 \mathrm{H}, \mathrm{CH}), 7.08-8.02(\mathrm{~m}, 6 \mathrm{H}, \mathrm{Ar}-\mathrm{H}) ;{ }^{13} \mathrm{C}$ NMR (DMSO- $\left.d_{6}\right) \delta$ : 20.2, 22.5, 27.1, 119.1, 123.4, 126.1, 127.1, 127.8, 129, 129.7, 133.1, 133.9, 138.2, 140.1, 152.2, 154.1; IR ( KBr, $\left.v_{\max }, \mathrm{cm}^{-1}\right)$ : 2223 (2 CN); MS (70 eV) m/z (\%): $312\left(\mathrm{M}^{+}, 10\right)$. Anal. calcd. for $\mathrm{C}_{20} \mathrm{H}_{16} \mathrm{~N}_{4}$ : C, 76.90; H, 5.16; N, 17.94; found C, 76.94; H, $5.10 ; \mathrm{N}, 17.90$.

Ethyl 2-cyano-2-[4-(2,4,6-trimethylphenyl)phthalazin1-yl]acetate (5a): Yield: $62 \%$; m.p. $215-216{ }^{\circ} \mathrm{C} ;{ }^{1} \mathrm{H}$ NMR $\left(\mathrm{DMSO}-d_{6}\right) \delta: 1.35\left(\mathrm{t}, J=10 \mathrm{~Hz}, 3 \mathrm{H}, \mathrm{CH}_{3}\right), 2.32\left(\mathrm{~s}, 3 \mathrm{H}, \mathrm{CH}_{3}\right)$, $2.50\left(\mathrm{~s}, 6 \mathrm{H}, 2 \mathrm{CH}_{3}\right), 4.31\left(\mathrm{q}, J=10 \mathrm{~Hz}, 2 \mathrm{H}, \mathrm{CH}_{2}\right), 5.03(\mathrm{~s}, 1 \mathrm{H}$, $\mathrm{CH}), 7.02-8(\mathrm{~m}, 6 \mathrm{H}, \mathrm{Ar}-\mathrm{H}) ;{ }^{13} \mathrm{C}$ NMR (DMSO- $\left.d_{6}\right) \delta: 15.2$, 20.3, 22.8, 37.2, 66.1,117.3, 123.8, 125.1, 127.3, 127.9, 129.5, $130.7,132.1,133.4,138.2,140.6,155.2,157.1,169.0$; IR $\left(\mathrm{KBr}, \mathrm{v}_{\max }, \mathrm{cm}^{-1}\right): 2231(\mathrm{CN}), 1735(\mathrm{CO}) ; \mathrm{MS}(70 \mathrm{eV}) \mathrm{m} / \mathrm{z}$ (\%): $359\left(\mathrm{M}^{+}, 20\right)$. Anal. calcd. for $\mathrm{C}_{22} \mathrm{H}_{21} \mathrm{~N}_{3} \mathrm{O}_{2}$ : C, 73.52; $\mathrm{H}$, 5.89; N, 11.69; found C, 73.50; H, 5.91; N, 11.66.

Ethyl 2-[4-(2,4,6-trimethylphenyl)phthalazin-1-yl]-3oxobutanoate (5b): Yield: $65 \%$; m.p. $188-189{ }^{\circ} \mathrm{C} ;{ }^{1} \mathrm{H}$ NMR $\left(\mathrm{DMSO}-d_{6}\right) \delta: 1.24\left(\mathrm{t}, J=10 \mathrm{~Hz}, 3 \mathrm{H}, \mathrm{CH}_{3}\right), 2.35\left(\mathrm{~s}, 3 \mathrm{H}, \mathrm{CH}_{3}\right)$, 2.50 (s, 6H, $\left.2 \mathrm{CH}_{3}\right), 2.61$ (s, 3H, $\left.\mathrm{COCH}_{3}\right), 4.44$ (q, $J=10 \mathrm{~Hz}$, $\left.2 \mathrm{H}, \mathrm{CH}_{2}\right), 5.11(\mathrm{~s}, 1 \mathrm{H}, \mathrm{CH}), 7.04-7.98(\mathrm{~m}, 6 \mathrm{H}, \mathrm{Ar}-\mathrm{H}) ;{ }^{13} \mathrm{C} \mathrm{NMR}$ $\left(\mathrm{DMSO}-d_{6}\right) \delta: 15.6,20.1,22,29.2,63.0,65.6,123,125.2$, $126.8,127.7,129,130.1,132.7,133,137.2,140,155.9,158.1$, 167.0, 188.1; IR (KBr, $\left.v_{\max }, \mathrm{cm}^{-1}\right)$ : 1710, 1735 (2 CO); MS $(70 \mathrm{eV}) \mathrm{m} / \mathrm{z}(\%): 376\left(\mathrm{M}^{+}, 9\right)$. Anal. calcd. for $\mathrm{C}_{23} \mathrm{H}_{24} \mathrm{~N}_{2} \mathrm{O}_{3}$ : C, 73.38; H, 6.43; N, 7.44; found C, 73.30; H, 6.47; N, 7.45.

3-[4-(2,4,6-Trimethylphenyl)phthalazin-1-yl]pentane2,4-dione (6): Yield: $64 \%$; m.p. $163-164{ }^{\circ} \mathrm{C} ;{ }^{1} \mathrm{H}$ NMR $\left(\mathrm{DMSO}-d_{6}\right) \delta: 2.31\left(\mathrm{~s}, 3 \mathrm{H}, \mathrm{CH}_{3}\right), 2.47\left(\mathrm{~s}, 6 \mathrm{H}, 2 \mathrm{CH}_{3}\right), 2.70(\mathrm{~s}$, $\left.6 \mathrm{H}, 2 \mathrm{COCH}_{3}\right), 5.01$ (s, 1H, CH), 7.01-7.99 (m, 6H, Ar-H); ${ }^{13} \mathrm{C}$ NMR (DMSO- $\left.d_{6}\right) \delta: 20.5,22.4,30.1,77.9,123.3,125.4,126.9$, 128.5, 129.1, 130.4, 132.7, 133.5, 136.5, 140.1, 155, 158, 190; IR (KBr, $\left.v_{\max }, \mathrm{cm}^{-1}\right): 1717$ (2 CO); MS (70 eV) $\mathrm{m} / \mathrm{z}(\%): 346$ $\left(\mathrm{M}^{+}, 18\right)$. Anal. calcd. for $\mathrm{C}_{22} \mathrm{H}_{22} \mathrm{~N}_{2} \mathrm{O}_{2}: \mathrm{C}, 76.28 ; \mathrm{H}, 6.40 ; \mathrm{N}$, 8.09; found C, 76.29; H, 6.46; N, 8.

General procedure for the synthesis of pyrazolylphthalazines 7-9: A mixture of 4, 5a, 5b, 6 ( $0.005 \mathrm{~mol})$ and hydrazine hydrate $(0.005 \mathrm{~mol})$ in absolute ethanol $(20 \mathrm{~mL})$ was heated under reflux for $6 \mathrm{~h}$ and then allowed to cool. The solid product was collected and recrystallized from the proper solvent to give the compounds 7-9, respectively.

4-[4-(2,4,6-Trimethylphenyl)phthalazin-1-yl]-4Hpyrazole-3,5-diamine (7): Yield: $62 \%$; m.p. $209-210{ }^{\circ} \mathrm{C} ;{ }^{1} \mathrm{H}$ NMR (DMSO-d $) \delta: 2.38\left(\mathrm{~s}, 3 \mathrm{H}, \mathrm{CH}_{3}\right), 2.51\left(\mathrm{~s}, 6 \mathrm{H}, 2 \mathrm{CH}_{3}\right)$, 4.11 (s, 1H, CH), 5.10-5.40 (br s, 4H, 2NH$), 7.04-8.01$ (m, $6 \mathrm{H}, \mathrm{Ar}-\mathrm{H}$ ); ${ }^{13} \mathrm{C}$ NMR (DMSO- $\left.d_{6}\right) \delta: 20.8,22.7,48.1,124$, 125.1, 126, 128.6, 129, 131, 132.4, 133.6, 136.5, 140.3, 154.5, 158.1, 170.2; IR $\left(\mathrm{KBr}, v_{\max }, \mathrm{cm}^{-1}\right): 3467-3296\left(\mathrm{NH}_{2}\right)$; MS (70 eV) $m / z$ (\%): $344\left(\mathbf{M}^{+}, 10\right)$. Anal. calcd. for $\mathrm{C}_{20} \mathrm{H}_{20} \mathrm{~N}_{6}$ : C, 69.75; H, 5.85; N, 24.40; found C, 69.71; H, 5.87; N, 24.44.

3-Amino-4-[4-(2,4,6-trimethylphenyl)phthalazin-1-yl]$\mathbf{1 H}$-pyrazol-5(4H)-one (8a): Yield: $59 \%$; m.p. $239-240{ }^{\circ} \mathrm{C}$; ${ }^{1} \mathrm{H}$ NMR (DMSO- $\left.d_{6}\right) \delta: 2.36$ (s, 3H, $\left.\mathrm{CH}_{3}\right), 2.50\left(\mathrm{~s}, 6 \mathrm{H}, 2 \mathrm{CH}_{3}\right)$, 4.22 (s, 1H, CH), 4.40 (br s, 2H, NH ${ }_{2}$ ), 6.92-8 (m, 7H, Ar-H + NH of pyrazole); ${ }^{13} \mathrm{C}$ NMR (DMSO- $d_{6}$ ) $\delta: 21,22.6,55.2,122.6$, 125, 126.3, 128.9, 129.7, 130.8, 132.8, 133, 136.6, 141, 155.4, 156.8, 159.1, 178.1; IR (KBr, $\left.v_{\max }, \mathrm{cm}^{-1}\right)$ : 3425-3211 (multiple bands, $\left.\mathrm{NH}_{2}, \mathrm{NH}\right), 1678(\mathrm{CO}), 1612(\mathrm{C}=\mathrm{N})$; MS $(70 \mathrm{eV}) \mathrm{m} / \mathrm{z}$ (\%): $345\left(\mathrm{M}^{+}, 8\right)$. Anal. calcd. for $\mathrm{C}_{20} \mathrm{H}_{19} \mathrm{~N}_{5} \mathrm{O}$ : C, 69.55; $\mathrm{H}$, 5.54; N, 20.28; found C, 69.50; H, 5.51; N, 20.30.

4-[4-(2,4,6-Trimethylphenyl)phthalazin-1-yl]-3methyl-1H-pyrazol-5(4H)-one (8b): Yield: $61 \%$; m.p. 250$251{ }^{\circ} \mathrm{C} ;{ }^{1} \mathrm{H}$ NMR (DMSO- $\left.d_{6}\right) \delta: 2.12\left(\mathrm{~s}, 3 \mathrm{H}, \mathrm{CH}_{3}\right), 2.38(\mathrm{~s}$, $\left.3 \mathrm{H}, \mathrm{CH}_{3}\right), 2.52\left(\mathrm{~s}, 6 \mathrm{H}, 2 \mathrm{CH}_{3}\right), 4.10(\mathrm{~s}, 1 \mathrm{H}, \mathrm{CH}), 6.96-8.09(\mathrm{~m}$, $7 \mathrm{H}, \mathrm{Ar}-\mathrm{H}+\mathrm{NH}$ of pyrazole); ${ }^{13} \mathrm{C}$ NMR (DMSO- $\left.d_{6}\right) \delta: 20.4$, 21.8, 22.5, 52.2, 123, 125, 127.4, 128, 129.5, 131.2, 132.4, 133.8, 136.8, 140.4, 152.1, 154.8, 158.1, 176.9; IR (KBr, $v_{\max }$, $\left.\mathrm{cm}^{-1}\right): 3224(\mathrm{NH}), 1679(\mathrm{CO}), 1611(\mathrm{C}=\mathrm{N})$; MS $(70 \mathrm{eV}) \mathrm{m} / \mathrm{z}$ (\%): $344\left(\mathrm{M}^{+}, 15\right)$. Anal. calcd. for $\mathrm{C}_{21} \mathrm{H}_{20} \mathrm{~N}_{4} \mathrm{O}: \mathrm{C}, 73.23 ; \mathrm{H}$, 5.85; N, 16.27; found C, 73.20; H, 5.87; N, 16.20.

1-(3,5-Dimethyl-1H-pyrazol-4-yl)-4-(2,4,6-trimethylphenyl)phthalazine (9): Yield: $60 \%$; m.p. 200-201 ${ }^{\circ} \mathrm{C} ;{ }^{1} \mathrm{H}$ NMR (DMSO- $\left.d_{6}\right) \delta: 2.34\left(\mathrm{~s}, 3 \mathrm{H}, \mathrm{CH}_{3}\right), 2.49\left(\mathrm{~s}, 6 \mathrm{H}, 2 \mathrm{CH}_{3}\right)$, $2.55,2.65\left(2 \mathrm{~s}, 6 \mathrm{H}, 2 \mathrm{CH}_{3}\right.$ of pyrazole $), 7.00-8.13(\mathrm{~m}, 7 \mathrm{H}$, $\mathrm{Ar}-\mathrm{H}+\mathrm{NH}$ of pyrazole); ${ }^{13} \mathrm{C}$ NMR (DMSO- $\left.d_{6}\right) \delta: 16.1,21$, 22.3, 120, 123.3, 125.3, 126.9, 128.8, 129, 131.5, 132.3, 133.7, 136.6, 140.4, 144, 144.6, 154.2, 157.2; IR (KBr, $\left.v_{\max }, \mathrm{cm}^{-1}\right)$ : $3211(\mathrm{NH}), 1609(\mathrm{C}=\mathrm{N})$; MS (70 eV) $\mathrm{m} / z(\%): 342\left(\mathrm{M}^{+}, 11\right)$. Anal. calcd. for $\mathrm{C}_{22} \mathrm{H}_{22} \mathrm{~N}_{4}$ : C, 77.16; H, 6.48; N, 16.36; found C, 77.19; H, 6.40; N, 16.37.

General procedure for the synthesis of pyrazolylphthalazine derivatives 10-13: A solution of $3(0.01 \mathrm{~mol})$ and active methylene compounds, namely ethyl acetoacetate, ethyl cyanoacetate, diethylmalonate, acetylacetone (0.012 mol), in ethanol $(25 \mathrm{~mL})$ containing catalytic amount of piperidine $(1 \mathrm{~mL})$ was refluxed for $4 \mathrm{~h}$. The solid compound obtained after cooling was crystallized from a suitable solvent to give the compounds 10-13.

1-[4-(2,4,6-Trimethylphenyl)phthalazin-1-yl]-3methyl-1H-pyrazol-5(4H)-one (10): Yield: $71 \%$; m.p. 270$271{ }^{\circ} \mathrm{C} ;{ }^{1} \mathrm{H}$ NMR (DMSO- $\left.d_{6}\right) \delta: 2.30\left(\mathrm{~s}, 3 \mathrm{H}, \mathrm{CH}_{3}\right), 2.47(\mathrm{~s}$, $\left.6 \mathrm{H}, 2 \mathrm{CH}_{3}\right), 2.61\left(\mathrm{~s}, 3 \mathrm{H}, \mathrm{CH}_{3}\right), 3.83\left(\mathrm{~s}, 2 \mathrm{H}, \mathrm{CH}_{2}\right), 7.01-8.03$ $(\mathrm{m}, 6 \mathrm{H}, \mathrm{Ar}-\mathrm{H}) ;{ }^{13} \mathrm{C}$ NMR (DMSO- $\left.d_{6}\right) \delta: 18.2,21.8,23,45.5$, $117.1,121.3,126,128.3,130.2,130.9,132,133.4,136.2$, 139.3, 147, 153.5, 161.3, 175.1; IR (KBr, $\left.v_{\max }, \mathrm{cm}^{-1}\right): 3246$ (NH), $1709(\mathrm{CO}), 1615(\mathrm{C}=\mathrm{N})$; MS (70 eV) $\mathrm{m} / \mathrm{z}(\%): 344\left(\mathrm{M}^{+}\right.$, 19). Anal. calcd. for $\mathrm{C}_{21} \mathrm{H}_{20} \mathrm{~N}_{4} \mathrm{O}: \mathrm{C}, 73.23 ; \mathrm{H}, 5.85 ; \mathrm{N}, 16.27$; found C, 73.20; H, 5.83; N, 16.29. 
3-Amino-1-[4-(2,4,6-trimethylphenyl)phthalazin-1-yl]$\mathbf{1 H}$-pyrazol-5(4H)-one (11): Yield: $68 \%$; m.p. 223-224 ${ }^{\circ} \mathrm{C}$; ${ }^{1} \mathrm{H}$ NMR (DMSO- $\left.d_{6}\right) \delta: 2.33\left(\mathrm{~s}, 3 \mathrm{H}, \mathrm{CH}_{3}\right), 2.49\left(\mathrm{~s}, 6 \mathrm{H}, 2 \mathrm{CH}_{3}\right)$, 3.42 (s, 2H, $\mathrm{CH}_{2}$ ), 4.47 (br s, $\left.2 \mathrm{H}, \mathrm{NH}_{2}\right), 7.04-8.04(\mathrm{~m}, 6 \mathrm{H}$, Ar-H) $;{ }^{13} \mathrm{C}$ NMR (DMSO- $\left.d_{6}\right) \delta: 21.3,22.9,75.2,116.6,121$, 126.4, 129.3, 130.5, 130.9, 132.2, 133.5, 136.1, 139.53, 147.1, 154.5, 167.1, 175.9; IR (KBr, $\left.v_{\max }, \mathrm{cm}^{-1}\right): 3379\left(\mathrm{NH}_{2}\right), 1712$ (CO), $1612(\mathrm{C}=\mathrm{N})$; MS (70 eV) $\mathrm{m} / \mathrm{z}(\%): 345\left(\mathrm{M}^{+}, 14\right)$. Anal. calcd. for $\mathrm{C}_{20} \mathrm{H}_{19} \mathrm{~N}_{5} \mathrm{O}$ : C, 69.55; H, 5.54; N, 20.28; found $\mathrm{C}$, 69.50; H, 5.58; N, 20.30.

1-[4-(2,4,6-Trimethylphenyl)phthalazin-1-yl]pyrazolidine-3,5-dione (12): Yield: $71 \%$; m.p. 236-237 ${ }^{\circ} \mathrm{C}$; ${ }^{1} \mathrm{H}$ NMR $\left(\mathrm{DMSO}-d_{6}\right) \delta: 2.33\left(\mathrm{~s}, 3 \mathrm{H}, \mathrm{CH}_{3}\right), 2.49\left(\mathrm{~s}, 6 \mathrm{H}, 2 \mathrm{CH}_{3}\right), 3.79(\mathrm{~s}$, 2H, $\left.\mathrm{CH}_{2}\right), 7.04-8.04$ (m, 6H, Ar-H), 9.88 (s, 1H, NH, exchangeable) ${ }^{13} \mathrm{C}$ NMR (DMSO- $\left.d_{6}\right) \delta: 20.8,23.1,50.1,116.7$, $121,126,129.1,130.4,130.9,132.1,133.5,137.1,139.2$, 147.1, 153.0, 166.1, 173.0; IR (KBr, $\left.v_{\max }, \mathrm{cm}^{-1}\right): 3224(\mathrm{NH})$, 1707-1666 (2CO), $1618(\mathrm{C}=\mathrm{N})$; MS (70 eV) $\mathrm{m} / \mathrm{z}(\%): 346$ $\left(\mathrm{M}^{+}, 7\right)$. Anal. calcd. for $\mathrm{C}_{20} \mathrm{H}_{18} \mathrm{~N}_{4} \mathrm{O}_{2}: \mathrm{C}, 69.35 ; \mathrm{H}, 5.24 ; \mathrm{N}$, 16.17; found C, 69.36; H, 5.29; N, 16.10.

1-(3,5-Dimethyl-1H-pyrazol-1-yl)-4-(2,4,6-trimethylphenyl)phthalazine (13): Yield: $70 \%$; m.p. $170-171{ }^{\circ} \mathrm{C} ;{ }^{1} \mathrm{H}$ NMR (DMSO-d $\left.d_{6}\right) \delta: 1.82\left(\mathrm{~s}, 3 \mathrm{H}, \mathrm{CH}_{3}\right), 2.35\left(\mathrm{~s}, 3 \mathrm{H}, \mathrm{CH}_{3}\right)$, $2.49\left(\mathrm{~s}, 6 \mathrm{H}, 2 \mathrm{CH}_{3}\right), 2.62\left(\mathrm{~s}, 3 \mathrm{H}, \mathrm{CH}_{3}\right), 6.13(\mathrm{~s}, 1 \mathrm{H}, \mathrm{CH}$ of pyrazole), 7.01-8.08 (m, 6H, Ar-H); ${ }^{13} \mathrm{C}$ NMR (DMSO- $d_{6}$ ) $\delta$ : 11.1, 15.3, 21.9, 23.2, 107.2, 118.1, 121.8, 126, 128.1, 130, 130.6, 131.1, 133.2, 136, 139, 142.2, 147.1, 152, 153.2; IR $\left(\mathrm{KBr}, v_{\max }, \mathrm{cm}^{-1}\right): 1610(\mathrm{C}=\mathrm{N}) ; \mathrm{MS}(70 \mathrm{eV}) \mathrm{m} / \mathrm{z}(\%): 342\left(\mathrm{M}^{+}\right.$, 17). Anal. calcd. for $\mathrm{C}_{22} \mathrm{H}_{22} \mathrm{~N}_{4}$ : C, 77.16; H, 6.48; N, 16.36; found $\mathrm{C}, 77.10 ; \mathrm{H}, 6.50 ; \mathrm{N}, 16.32$.

3-[2-(4-(2,4,6-Trimethylphenyl)phthalazin-1-yl]hydrazinyl)propanenitrile (14): A solution of $3(0.01 \mathrm{~mol})$ and acrylonitrile $(0.01 \mathrm{~mol})$ was refluxed in pyridine $(25 \mathrm{~mL})$ for $4 \mathrm{~h}$. After cooling, the reaction mixture was poured onto ice and $\mathrm{HCl}$ and the solid formed was crystallized from benzene to give 14. Yield: $66 \%$; m.p. $280-281{ }^{\circ} \mathrm{C}$; ${ }^{1} \mathrm{H}$ NMR (DMSO- $d_{6}$ ) $\delta: 2.10\left(\mathrm{~s}, 1 \mathrm{H}, \mathrm{NHCH}_{2}\right.$, exchangeable with $\left.\mathrm{D}_{2} \mathrm{O}\right), 2.36(\mathrm{~s}, 3 \mathrm{H}$, $\left.\mathrm{CH}_{3}\right), 2.48\left(\mathrm{~s}, 6 \mathrm{H}, 2 \mathrm{CH}_{3}\right), 3.12\left(\mathrm{~s}, 2 \mathrm{H}, \mathrm{CH}_{2}\right), 3.45\left(\mathrm{~s}, 2 \mathrm{H}, \mathrm{CH}_{2}\right)$, 7.02-8.02 (m, 6H, Ar-H) 8.11 (s, 1H, NH, exchangeable with $\left.\mathrm{D}_{2} \mathrm{O}\right) ;{ }^{13} \mathrm{C}$ NMR (DMSO- $d_{6}$ ) $\delta: 17.1,21.3,23.2,48.5,117.2$, 120.1, 121.0, 127.1, 129.4, 130.7, 131.5, 132, 133.5, 136.2, 139.9, 148, 157.1; IR (KBr, $\left.v_{\max }, \mathrm{cm}^{-1}\right): 3290-3210(\mathrm{NH}), 2221$ (CN), $1616(\mathrm{C}=\mathrm{N})$; MS (70 eV) m/z (\%): $331\left(\mathrm{M}^{+}, 6\right)$. Anal. calcd. for $\mathrm{C}_{20} \mathrm{H}_{21} \mathrm{~N}_{5}$ : C, 72.48; H, 6.39; N, 21.13; found C, 72.42; $\mathrm{H}, 6.34 ; \mathrm{N}, 21.17$.

2-[4-(2,4,6-Trimethylphenyl)phthalazin-1-yl]-2,5dihydro-1H-pyrazol-3-amine (15): A solution of $\mathbf{1 4}(0.01$ $\mathrm{mol})$ in ethanol $(25 \mathrm{~mL})$ and sodium hydroxide $(15 \mathrm{~mL}, 20 \%)$ was refluxed for $8 \mathrm{~h}$. The reaction mixture was cooled to room temperature and acidified with hydrochloric acid to give a solid product which was crystallized to give $\mathbf{1 5}$. Yield: $51 \%$; m.p. 208-209 ${ }^{\circ} \mathrm{C}$; ${ }^{1} \mathrm{H}$ NMR (DMSO- $d_{6}$ ) $\delta: 2.10$ (s, $1 \mathrm{H}, \mathrm{NHCH}_{2}$, exchangeable with $\left.\mathrm{D}_{2} \mathrm{O}\right), 2.33\left(\mathrm{~s}, 3 \mathrm{H}, \mathrm{CH}_{3}\right), 2.49\left(\mathrm{~s}, 6 \mathrm{H}, 2 \mathrm{CH}_{3}\right)$, $4.10\left(\mathrm{t}, J=7.1 \mathrm{~Hz}, 2 \mathrm{H}, \mathrm{CH}_{2}\right.$ of pyrazol moiety), 4.55 (d, $J=$ $7.1 \mathrm{~Hz}, 1 \mathrm{H}$, methine proton of pyrazole), 6.23 (br s, $2 \mathrm{H}, \mathrm{NH}_{2}$ ), 7.01-8 (m, 6H, Ar-H) $9.91(\mathrm{~s}, 1 \mathrm{H}, \mathrm{NH}$, exchangeable with $\mathrm{D}_{2} \mathrm{O}$ ); ${ }^{13} \mathrm{C}$ NMR (DMSO- $d_{6}$ ) $\delta: 21.5,23.4,48.7,80.8,117.4$, 121.0, 126.1, 128.3, 130.3, 130.8, 131.4, 133.5, 136.2, 139.5,
146, 153.6, 159; IR (KBr, $\left.v_{\max }, \mathrm{cm}^{-1}\right): 3380-3150(\mathrm{NH}$ and $\left.\mathrm{NH}_{2}\right), 1615(\mathrm{C}=\mathrm{N})$; MS $(70 \mathrm{eV}) \mathrm{m} / z(\%): 331\left(\mathrm{M}^{+}, 19\right)$. Anal. calcd. for $\mathrm{C}_{20} \mathrm{H}_{21} \mathrm{~N}_{5}$ : C, 72.48; H, 6.39; N, 21.13; found $\mathrm{C}$, 72.48; H, 6.41; N, 21.15.

General procedure for the synthesis of pyrazolylphthalazine derivatives 16-19: To a solution of hydrazinophthalazine $3(0.01 \mathrm{~mol})$ in $(30 \mathrm{~mL})$ anhydrous ethanol, ethoxymethylenemalononitrile, tetracyanoethylene, ethyl (ethoxymethylene)cyanoacetate, or methyl bis(methylthio)ethoxymethylene cyanoacetate $(0.01 \mathrm{~mol})$ was added and the reaction mixtures were refluxed for 3-5 h, respectively. The products, which separated on cooling, were collected by filtration and recrystallized from a suitable solvent to give compounds 16-19.

5-Amino-1-[4-(2,4,6-trimethylphenyl)phthalazin-1-yl]$1 \mathrm{H}$-pyrazole-4-carbonitrile (16): Yield: $88 \%$; m.p. $261-262{ }^{\circ} \mathrm{C}$; ${ }^{1} \mathrm{H}$ NMR (DMSO- $\left.d_{6}\right) \delta: 2.36\left(\mathrm{~s}, 3 \mathrm{H}, \mathrm{CH}_{3}\right), 2.50$ (s, 6H, 2CH $\mathrm{CH}_{3}$, 6.88 (br s, $2 \mathrm{H}, \mathrm{NH}_{2}$ exchangeable with $\mathrm{D}_{2} \mathrm{O}$ ), 7.05-8.03 (m, $6 \mathrm{H}, \mathrm{Ar}-\mathrm{H}), 8.21$ (s, 1H, CH of pyrazole); ${ }^{13} \mathrm{C}$ NMR (DMSO$\left.d_{6}\right) \delta: 21.4,23.1,72,116.1,122,125.3,125.9,126.3,128$, 129.1, 134.1, 134.9, 137.2, 139, 145.8, 152, 155.4, 165.3; IR $\left(\mathrm{KBr}, \mathrm{v}_{\max }, \mathrm{cm}^{-1}\right): 3407,3204\left(\mathrm{NH}_{2}\right), 2212(\mathrm{CN}), 1605(\mathrm{C}=\mathrm{N})$; MS (70 eV) $m / z(\%): 354\left(\mathrm{M}^{+}, 10\right)$. Anal. calcd. for $\mathrm{C}_{21} \mathrm{H}_{18} \mathrm{~N}_{6}$ : C, 71.17; H, 5.12; N, 23.71; found C, 71.10; H, 5.17; N, 23.75.

5-Amino-1-[4-(2,4,6-trimethylphenyl)phthalazin-1-yl]$1 H$-pyrazole-3,4-dicarbonitrile (17): Yield: $65 \%$; m.p. 216$217{ }^{\circ} \mathrm{C} ;{ }^{1} \mathrm{H}$ NMR (DMSO- $\left.d_{6}\right) \delta: 2.32\left(\mathrm{~s}, 3 \mathrm{H}, \mathrm{CH}_{3}\right), 2.47$ (s, $6 \mathrm{H}, 2 \mathrm{CH}_{3}$ ), 6.92 (br s, $2 \mathrm{H}, \mathrm{NH}_{2}$ exchangeable with $\mathrm{D}_{2} \mathrm{O}$ ), 7.05$8.04(\mathrm{~m}, 6 \mathrm{H}, \mathrm{Ar}-\mathrm{H}) ;{ }^{13} \mathrm{C}$ NMR (DMSO- $\left.d_{6}\right) \delta: 21.1,23,89.1$, 114.1, 116.7, 120.1, 122.1, 125.4, 126, 126.8, 127.3, 129.3, $134.5,134.9,137.5,140,152.1,155.5,162.2$; IR (KBr, $v_{\max }$, $\left.\mathrm{cm}^{-1}\right)$ : 3411, $3202\left(\mathrm{NH}_{2}\right), 2224(\mathrm{CN}), 1610(\mathrm{C}=\mathrm{N})$; MS $(70$ eV) $m / z(\%): 379\left(\mathbf{M}^{+}, 8\right)$. Anal. calcd. for $\mathrm{C}_{22} \mathrm{H}_{17} \mathrm{~N}_{7}$ : C, 69.64; $\mathrm{H}, 4.52 ; \mathrm{N}, 25.84$; found $\mathrm{C}, 69.64 ; \mathrm{H}, 4.52 ; \mathrm{N}, 25.84$.

Ethyl 5-amino-1-[4-(2,4,6-trimethylphenyl)phthalazin1-yl]-1H-pyrazole-4-carboxylate (18): Yield: $85 \%$; m.p. 200$201{ }^{\circ} \mathrm{C} ;{ }^{1} \mathrm{H}$ NMR (DMSO- $\left.d_{6}\right) \delta: 1.35\left(\mathrm{t}, J=6.9 \mathrm{~Hz}, 3 \mathrm{H}, \mathrm{CH}_{3}\right.$ ), $2.34\left(\mathrm{~s}, 3 \mathrm{H}, \mathrm{CH}_{3}\right), 2.47\left(\mathrm{~s}, 6 \mathrm{H}, 2 \mathrm{CH}_{3}\right), 4.22(\mathrm{q}, J=6.9 \mathrm{~Hz}, 2 \mathrm{H}$, $\mathrm{CH}_{2}$ ), 6.94 (br s, $2 \mathrm{H}, \mathrm{NH}_{2}$ exchangeable with $\mathrm{D}_{2} \mathrm{O}$ ), 7.02-8.16 (m, 7H, Ar-H + CH of pyrazole); ${ }^{13} \mathrm{C}$ NMR (DMSO- $\left.d_{6}\right) \delta$ : 15.3, 21.4, 23.1, 55.3, 109.1, 121.6, 125.1, 126, 126.7, 127.3, 128.3, 133.1, 134.2, 134.9, 137, 139, 152, 155.1, 160.1, 167.8; IR $\left(\mathrm{KBr}, v_{\max }, \mathrm{cm}^{-1}\right): 3464,3354\left(\mathrm{NH}_{2}\right), 1729(\mathrm{CO}), 1605$ $(\mathrm{C}=\mathrm{N})$; MS $(70 \mathrm{eV}) \mathrm{m} / \mathrm{z}(\%)$ : $401\left(\mathrm{M}^{+}, 14\right)$. Anal. calcd. for $\mathrm{C}_{23} \mathrm{H}_{23} \mathrm{~N}_{5} \mathrm{O}_{2}$ : C, 68.81; H, 5.77; N, 17.44; found $\mathrm{C}, 68.79 ; \mathrm{H}$, 5.770; N, 17.47 .

Methyl 5-amino-1-[4-(2,4,6-trimethylphenyl)phthalazin-1-yl]-3-(methylthio)-1 $\boldsymbol{H}$-pyrazole-4-carboxylate (19): Yield: $87 \%$; m.p. $221-222{ }^{\circ} \mathrm{C} ;{ }^{1} \mathrm{H}$ NMR (DMSO- $d_{6}$ ) $\delta$ : 2.37 (s, 3H, $\left.\mathrm{CH}_{3}\right), 2.50\left(\mathrm{~s}, 6 \mathrm{H}, 2 \mathrm{CH}_{3}\right), 3.81$ (s, 3H, $\left.\mathrm{SCH}_{3}\right)$, $4.01\left(\mathrm{~s}, 3 \mathrm{H}, \mathrm{COOCH}_{3}\right), 6.90$ (br s, $2 \mathrm{H}, \mathrm{NH}_{2}$ exchangeable with $\left.\mathrm{D}_{2} \mathrm{O}\right), 7.01-8(\mathrm{~m}, 6 \mathrm{H}, \mathrm{Ar}-\mathrm{H}) ;{ }^{13} \mathrm{C}$ NMR (DMSO- $\left.d_{6}\right) \delta: 14.5$, 21.0, 22.3, 55.1, 99, 122, 125.3, 126.1, 126.9, 127, 129, 134.6, $134.9,137.1,140.1,143.1,152.3,155.9,159.4,169.1$; IR $\left(\mathrm{KBr}, \mathrm{v}_{\max }, \mathrm{cm}^{-1}\right): 3410,3233\left(\mathrm{NH}_{2}\right), 1731(\mathrm{CO}), 1611(\mathrm{C}=\mathrm{N})$; MS (70 eV) m/z (\%): $447\left(\mathrm{M}^{+}, 5\right)$. Anal. calcd. for $\mathrm{C}_{24} \mathrm{H}_{25} \mathrm{~N}_{5} \mathrm{O}_{2} \mathrm{~S}$ : C, 64.41; H, 5.63; N, 15.65; S, 7.16; found C, 64.40; H, 5.68; N, 15.66; S, 7.10. 
General procedure for the synthesis of sugar hydrazone derivatives 20 a-e: To a solution of hydrazinophthalazine $\mathbf{3}$ $(0.1 \mathrm{~mol})$ in ethanol $(50 \mathrm{~mL})$ was added the respective sugar $(0.1 \mathrm{~mol})$ and catalytic amount of glacial acetic acid $(0.1 \mathrm{~mL})$. The mixture was heated under reflux on a water bath for $5 \mathrm{~h}$. After cooling the separated solid was collected by filtration, dried and crystallized from the appropriate solvent to give $\mathbf{2 0}$ a-e.

D-Glucose [4-(2,4,6-trimethylphenyl)phthalazine-1yl]-hydrazone (20a): Yield: $60 \%$; m.p. 200-201 ${ }^{\circ} \mathrm{C}$; ${ }^{1} \mathrm{H}$ NMR $\left(\mathrm{DMSO}-d_{6}\right) \delta: 2.35\left(\mathrm{~s}, 3 \mathrm{H}, \mathrm{CH}_{3}\right), 2.51\left(\mathrm{~s}, 6 \mathrm{H}, 2 \mathrm{CH}_{3}\right), 3.21-3.60$ (protons of the alditol congregated with the solvent absorption), 3.72-3.85 (m, 2H, $\left.\mathrm{CH}_{2} \mathrm{OH}\right), 4.42-5.12\left(\mathrm{~m}, 5 \mathrm{H}, 5 \mathrm{OH}, \mathrm{D}_{2} \mathrm{O}\right.$ exchangeable), $6.77(\mathrm{~d}, 1 \mathrm{H}, \mathrm{N}=\mathrm{CH}), 7.01-8.11(\mathrm{~m}, 7 \mathrm{H}, \mathrm{Ar}-\mathrm{H}$ and $\mathrm{NH}) ;{ }^{13} \mathrm{C}$ NMR (DMSO- $d_{6}$ ) $\delta: 21.1,22.9,65.5,66.3,71.2$, 73.0, 73.8, 117, 118.3, 127.1, 127.5, 129, 129.8, 132.1, 133.2, 137.1, 139.1, 144.1, 154.1, 165.7; IR (KBr, $\left.v_{\max }, \mathrm{cm}^{-1}\right): 3418-$ $3211(\mathrm{OH}, \mathrm{NH}), 1618(\mathrm{C}=\mathrm{N})$; MS (70 eV) m/z (\%): $440\left(\mathrm{M}^{+}\right.$, 6). Anal. calcd. for $\mathrm{C}_{23} \mathrm{H}_{28} \mathrm{~N}_{4} \mathrm{O}_{5}$ : C, 62.71; H, 6.41; N, 12.72; found $\mathrm{C}, 62.70 ; \mathrm{H}, 6.45 ; \mathrm{N}, 12.70$.

D-Galactose [4-(2,4,6-trimethylphenyl)phthalazine-1yl]-hydrazone (20b): Yield: $91 \%$; m.p. 181-182 ${ }^{\circ} \mathrm{C} ;{ }^{1} \mathrm{H}$ NMR $\left(\mathrm{DMSO}-d_{6}\right) \delta: 2.33\left(\mathrm{~s}, 3 \mathrm{H}, \mathrm{CH}_{3}\right), 2.51\left(\mathrm{~s}, 6 \mathrm{H}, 2 \mathrm{CH}_{3}\right), 3.20-$ 3.66 (protons of the alditol congregated with the solvent absorption), 3.70-3.81 (m, 2H, $\left.\mathrm{CH}_{2} \mathrm{OH}\right), 4.41-5.17$ (m, 5H, 5OH, $\mathrm{D}_{2} \mathrm{O}$ exchangeable), $6.70(\mathrm{~d}, 1 \mathrm{H}, \mathrm{N}=\mathrm{CH}), 7.04-8.09(\mathrm{~m}$, $7 \mathrm{H}, \mathrm{Ar}-\mathrm{H}$ and $\mathrm{NH}$ ); ${ }^{13} \mathrm{C}$ NMR (DMSO- $\left.d_{6}\right) \delta: 21,22.8,65.1$, 67.1, 72, 73.5, 73.9, 118, 118.9, 127.5, 127.9, 129, 129.7, 132.1, 133, 137, 139.6, 144, 153.1, 165; IR (KBr, $\left.v_{\max }, \mathrm{cm}^{-1}\right)$ : 3433-3234 (OH, NH), $1621(\mathrm{C}=\mathrm{N})$. Anal. calcd. for $\mathrm{C}_{23} \mathrm{H}_{28} \mathrm{~N}_{4} \mathrm{O}_{5}$ : C, 62.71; H, 6.41; N, 12.72; found C, 62.75; H, 6.40; N, 12.75 .

D-Mannose [4-(2,4,6-trimethylphenyl)phthalazine-1yl]-hydrazone (20c): Yield: $78 \%$; m.p. $213-214{ }^{\circ} \mathrm{C} ;{ }^{1} \mathrm{H}$ NMR $\left(\mathrm{DMSO}-d_{6}\right) \delta: 2.30\left(\mathrm{~s}, 3 \mathrm{H}, \mathrm{CH}_{3}\right), 2.46\left(\mathrm{~s}, 6 \mathrm{H}, 2 \mathrm{CH}_{3}\right), 3.28-3.67$ (protons of the alditol congregated with the solvent absorption), 3.75-3.81 (m, 2H, $\left.\mathrm{CH}_{2} \mathrm{OH}\right), 4.38-5.07\left(\mathrm{~m}, 5 \mathrm{H}, 5 \mathrm{OH}, \mathrm{D}_{2} \mathrm{O}\right.$ exchangeable), $6.75(\mathrm{~d}, 1 \mathrm{H}, \mathrm{N}=\mathrm{CH}), 7.05-8.04(\mathrm{~m}, 7 \mathrm{H}, \mathrm{Ar}-\mathrm{H}$ and NH); ${ }^{13} \mathrm{C}$ NMR (DMSO- $\left.d_{6}\right) \delta: 20.2,22.6,65.2,66.5,71.2$, 73.5, 73.9, 117.4, 118.8, 127.1, 128.5, 129, 130.8, 132.1, 134.2, 137.1, 140.1, 144.2, 154.6, 166.7; IR (KBr, $\left.v_{\max }, \mathrm{cm}^{-1}\right)$ : 3409$3210(\mathrm{OH}, \mathrm{NH}), 1617(\mathrm{C}=\mathrm{N})$. Anal. calcd. for $\mathrm{C}_{23} \mathrm{H}_{28} \mathrm{~N}_{4} \mathrm{O}_{5}: \mathrm{C}$, $62.71 ; \mathrm{H}, 6.41 ; \mathrm{N}, 12.72$; found $\mathrm{C}, 62.70 ; \mathrm{H}, 6.48 ; \mathrm{N}, 12.72$.

D-Xylose [4-(2,4,6-trimethylphenyl)phthalazine-1-yl]hydrazone (20d): Yield: $59 \%$; m.p. $207-208{ }^{\circ} \mathrm{C}$; ${ }^{1} \mathrm{H}$ NMR $\left(\mathrm{DMSO}-d_{6}\right) \delta: 2.30\left(\mathrm{~s}, 3 \mathrm{H}, \mathrm{CH}_{3}\right), 2.46\left(\mathrm{~s}, 6 \mathrm{H}, 2 \mathrm{CH}_{3}\right), 3.34-$ 3.51 (protons of the alditol congregated with the solvent absorption), 3.61-3.73 (m, 2H, $\left.\mathrm{CH}_{2} \mathrm{OH}\right)$, 4.20-5.91 (m, 4H, 4OH, $\mathrm{D}_{2} \mathrm{O}$ exchangeable), $6.69(\mathrm{~d}, 1 \mathrm{H}, \mathrm{N}=\mathrm{CH}), 7-8.07(\mathrm{~m}, 7 \mathrm{H}, \mathrm{Ar}-$ $\mathrm{H}$ and $\mathrm{NH}$ ); ${ }^{13} \mathrm{C}$ NMR (DMSO- $\left.d_{6}\right) \delta: 20.8,22.9,65.1,66.9$, 73.2, 75.6, 116.1, 118, 126.2, 128, 129.2, 130.9, 131.1, 133.1, 136.1, 141.1, 144.1, 156.6, 168.3; IR ( $\left.\mathrm{KBr}, \mathrm{v}_{\max }, \mathrm{cm}^{-1}\right)$ : 3438$3222(\mathrm{OH}, \mathrm{NH}), 1617(\mathrm{C}=\mathrm{N})$. Anal. calcd. for $\mathrm{C}_{22} \mathrm{H}_{26} \mathrm{~N}_{4} \mathrm{O}_{4}: \mathrm{C}$, 64.37; H, 6.38; N, 13.65; found C, 64.30; H, 6.40; N, 13.60 .

D-Arabinose [4-(2,4,6-trimethylphenyl)phthalazine-1yl]-hydrazone (20e): Yield: $62 \%$; m.p. $179-180{ }^{\circ} \mathrm{C} ;{ }^{1} \mathrm{H}$ NMR $\left(\mathrm{DMSO}-d_{6}\right) \delta: 2.32$ (s, 3H, $\left.\mathrm{CH}_{3}\right), 2.49$ (s, 6H, 2CH 3 ), 3.323.48 (protons of the alditol congregated with the solvent absorption), 3.60-3.71 (m, 2H, $\left.\mathrm{CH}_{2} \mathrm{OH}\right), 4.21-5.94(\mathrm{~m}, 4 \mathrm{H}$, $4 \mathrm{OH}, \mathrm{D}_{2} \mathrm{O}$ exchangeable), $6.66(\mathrm{~d}, 1 \mathrm{H}, \mathrm{N}=\mathrm{CH}), 7.02-8.10(\mathrm{~m}$, $7 \mathrm{H}, \mathrm{Ar}-\mathrm{H}$ and NH); ${ }^{13} \mathrm{C}$ NMR (DMSO- $d_{6}$ ) $\delta: 20.4,23.2,65.4$,
66.7, 74.2, 75.3, 117.1, 118, 126.5, 128.4, 129.4, 131.2, 131.8, 133, 137.1, 140.1, 144.1, 156, 167.5; IR (KBr, $\left.v_{\max }, \mathrm{cm}^{-1}\right)$ : 3430-3205 (OH, NH), $1617(\mathrm{C}=\mathrm{N})$; MS (70 eV) $\mathrm{m} / \mathrm{z}(\%): 410$ $\left(\mathrm{M}^{+}, 6\right)$. Anal. calcd. for $\mathrm{C}_{22} \mathrm{H}_{26} \mathrm{~N}_{4} \mathrm{O}_{4}: \mathrm{C}, 64.37 ; \mathrm{H}, 6.38 ; \mathrm{N}$, 13.65; found C, 64.32; H, 6.44; N, 13.61.

General procedure for synthesis of per- $O$-acetyl-sugar hydrazone derivatives (21 a-e): A cold solution of $\mathbf{2 0}$ a-e (0.02 mol) in pyridine $(50 \mathrm{~mL})$ was treated with acetic anhydride $(60 \mathrm{~mL})$. The mixture was kept overnight at room temperature with occasional shaking. The mixture was poured onto ice- $\mathrm{H}_{2} \mathrm{O}$ and the product was collected by filtration, washed repeatedly with water, dried and recrystallized from ethanol to give compounds 21 a-e.

2,3,4,5,6-Penta- $O$-acetyl-D-glucose[1-acetyl-1- $\{4$ (2,4,6-trimethylphenyl)phthalazine-1-yl\}-hydrazone] (21a): Yield: $58 \%$; m.p. $60-61{ }^{\circ} \mathrm{C}$; ${ }^{1} \mathrm{H}$ NMR (DMSO- $\left.d_{6}\right) \delta$ : 2.02, 2.04, 2.10 (3s, $15 \mathrm{H}, 5 \mathrm{O} \mathrm{Ac}), 2.35$ (s, 3H, $\left.\mathrm{CH}_{3}\right), 2.46$ (s, $\left.6 \mathrm{H}, 2 \mathrm{CH}_{3}\right), 2.53$ (s, 3H, NAc), 4.15 (dd, 1H, H-6'), 4.26 (dd, 1H, H-6), 5.02-5.10 (m, 1H, H-5), 5.44-5.55 (m, 2H, H-4, H3), $5.62(\mathrm{dd}, 1 \mathrm{H}, \mathrm{H}-2), 6.74(\mathrm{~d}, 1 \mathrm{H}, \mathrm{N}=\mathrm{CH}), 7.08-8.11(\mathrm{~m}$, $6 \mathrm{H}, \mathrm{Ar}-\mathrm{H}) ;{ }^{13} \mathrm{C}$ NMR (DMSO- $\left.d_{6}\right) \delta: 20,20.8,21.3,22.1,22.9$, 62.2, 66.9, 68.6, 70.9, 72, 118, 118.5, 126.1, 127.2, 128.1, 129.3, 132, 133.1, 137.1, 139, 143.1, 154.1, 165.1, 174.2, 177.0; IR (KBr, $v_{\max }, \mathrm{cm}^{-1}$ ): 1719 (OAc), 1674 (NAc), 1608 $(\mathrm{C}=\mathrm{N})$. Anal. calcd. for $\mathrm{C}_{25} \mathrm{H}_{30} \mathrm{~N}_{4} \mathrm{O}_{6}: \mathrm{C}, 62.23 ; \mathrm{H}, 6.27 ; \mathrm{N}$, 11.61; found C, 62.29; H, 6.25; N, 11.60.

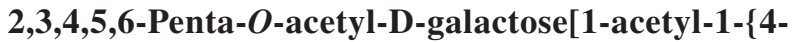
(2,4,6-trimethylphenyl)phthalazine-1-yl\}-hydrazone] (21b): Yield: $80 \%$; m.p. $161-162{ }^{\circ} \mathrm{C} ;{ }^{1} \mathrm{H}$ NMR (DMSO- $\left.d_{6}\right) \delta$ : 1.96, 1.99, 2.03, 2.08, 2.09 (5s, 15H, $5 \mathrm{O} \mathrm{Ac}), 2.30$ (s, 3H, $\mathrm{CH}_{3}$ ), 2.48 (s, 6H, 2CH3), 2.55(s, 3H, NAc), 3.88(dd, 1H, H6'), 4.28 (dd, 1H, H-6), 5.38-5.88 (m, 4H, H-5, H-4, H-3, H2), 6.55 (d, 1H, N=CH), 7.03-8.10 (m, 6H, Ar-H); ${ }^{13} \mathrm{C}$ NMR $\left(\mathrm{DMSO}-d_{6}\right) \delta$ : 19.6, 20.6, 21.4, 22.3, 22.8, 62, 66.5, 68.6, 70.7, 72.0, 118.1, 118.9, 126, 127.1, 128.1, 128.9, 132, 133.2, 137.1, 139.4, 144.6, 154.2, 165.8, 172.9, 176.8; IR (KBr, $\left.v_{\max }, \mathrm{cm}^{-1}\right)$ : 1723 (OAc), 1677 (NAc), $1611(\mathrm{C}=\mathrm{N})$; MS (70 eV) $\mathrm{m} / z(\%)$ : $482\left(\mathrm{M}^{+}, 8\right)$. Anal. calcd for $\mathrm{C}_{25} \mathrm{H}_{30} \mathrm{~N}_{4} \mathrm{O}_{6}: \mathrm{C}, 62.23 ; \mathrm{H}, 6.27 ; \mathrm{N}$, 11.61; found C, 62.30; H, 6.20; N, 11.64.

2,3,4,5,6-Penta- $O$-acetyl-D-mannose[1-acetyl-1- $\{4$ (2,4,6-trimethylphenyl)phthalazine-1-yl\}-hydrazone] (21c): Yield: $63 \%$; m.p. $58-60{ }^{\circ} \mathrm{C}$; ${ }^{1} \mathrm{H}$ NMR (DMSO-d6) $\delta: 2.03$, 2.06, 2.11 (3s, 15H, $5 \mathrm{OAc}), 2.36\left(\mathrm{~s}, 3 \mathrm{H}, \mathrm{CH}_{3}\right), 2.49$ (s, 6H, $2 \mathrm{CH}_{3}$ ), 2.55 (s, 3H, NAc), 4.14 (dd, 1H, H-6'), 4.28 (dd, 1H, H-6), 5.22-5.40 (m, 1H, H-5), 5.42 (m, 2H, H-4), 5.54 (dd, $1 \mathrm{H}, \mathrm{H}-3), 5.66$ (dd, 1H, H-2), 6.68 (d, 1H, N=CH), 7.04-8.08 $(\mathrm{m}, 6 \mathrm{H}, \mathrm{Ar}-\mathrm{H}) ;{ }^{13} \mathrm{C}$ NMR (DMSO- $\left.d_{6}\right) \delta: 20.4,20.9,21.8,22.3$, 23.9, 62.1, 66.2, 68.3, 70.5, 72.6, 118.2, 118.7, 126.1, 127.2, 128.6, 130.3, 132.1, 133.4, 137.3, 139.2, 143.9, 154.2, 166.3, 173.3, 176.4; IR (KBr, $\left.v_{\max }, \mathrm{cm}^{-1}\right): 1713$ (OAc), 1682 (NAc), $1615(\mathrm{C}=\mathrm{N})$. Anal. calcd. for $\mathrm{C}_{25} \mathrm{H}_{30} \mathrm{~N}_{4} \mathrm{O}_{6}$ : C, 62.23; H, 6.27; $\mathrm{N}, 11.61$; found $\mathrm{C}, 62.25 ; \mathrm{H}, 6.24 ; \mathrm{N}, 11.66$.

2,3,4,5,6-Penta- $O$-acetyl-D-xylose[1-acetyl-1- $\{4-(2,4,6-$ trimethylphenyl)phthalazine-1-yl\}-hydrazone] (21d): Yield: $53 \%$; m.p. 97-98 ${ }^{\circ} \mathrm{C} ;{ }^{1} \mathrm{H}$ NMR (DMSO- $\left.d_{6}\right) \delta: 1.91,1.94,2$, 2.19 (4s, 12H, 4OAc), 2.35 (s, 3H, $\mathrm{CH}_{3}$ ), 2.47 (s, 6H, 2CH $\mathrm{CH}_{3}$, 2.51 (s, 3H, NAc), 4.22 (dd, 1H, H-5'), 4.38 (dd, 1H, H-5), 5.38-5.40 (m, 1H, H-4), 5.78 (m, 2H, H-3), 6.01 (dd, 1H, H2), $6.58(\mathrm{~d}, 1 \mathrm{H}, \mathrm{N}=\mathrm{CH}), 7-8.04(\mathrm{~m}, 6 \mathrm{H}, \mathrm{Ar}-\mathrm{H}) ;{ }^{13} \mathrm{C} \mathrm{NMR}$ 
$\left(\mathrm{DMSO}-d_{6}\right) \delta: 20.1,20.9,21.7,22.4,23.5,62.4,66.4,68.9$, 70, 116.2, 118.6, 126, 127.1, 128.4, 130, 132.1, 133.8, 138.3, 140.2, 143.7, 154, 166.8, 171.3, 175.4; IR (KBr, $\left.v_{\max }, \mathrm{cm}^{-1}\right)$ : 1726 (OAc), 1685 (NAc), $1610(\mathrm{C}=\mathrm{N})$. Anal. calcd. for $\mathrm{C}_{24} \mathrm{H}_{28} \mathrm{~N}_{4} \mathrm{O}_{5}$ : C, 63.70; H, 6.24; N, 12.38; found $\mathrm{C}, 63.65 ; \mathrm{H}$, $6.29 ; \mathrm{N}, 12.34$.

2,3,4,5,6-Penta- $O$-acetyl-D-arabinose[1-acetyl-1- $\{4$ (2,4,6-trimethylphenyl)phthalazine-1-yl \}hydrazone] (21e): Yield: $67 \%$; m.p. $110-111{ }^{\circ} \mathrm{C}$; ${ }^{1} \mathrm{H}$ NMR (DMSO- $\left.d_{6}\right) \delta: 1.90$, 1.93, 2.01, 2.19 (4s, 12H, 4OAc), 2.37 (s, 3H, $\left.\mathrm{CH}_{3}\right), 2.50$ (s, $\left.6 \mathrm{H}, 2 \mathrm{CH}_{3}\right), 2.54$ (s, 3H, NAc), 4.27 (dd, 1H, H-5'), 4.39 (dd, 1H, H-5), 5.39-5.45 (m, 1H, H-4), 5.79 (m, 2H, H-3), 6.11 (dd, 1H, H-2), 6.60 (d, 1H, N=CH), 7.01-8.09 (m, 6H, Ar-H); ${ }^{13} \mathrm{C}$ NMR (DMSO- $d_{6}$ ) $\delta: 20,20.8,21.9,22.4,23.7,62.7,66$, 68.1, 70.8, 116.2, 117.6, 126, 127, 128.4, 130.5, 131.8, 133.4, $138.3,140.1,144.7,154.2,166,171.4,176.4$; IR (KBr, $v_{\max }$, $\left.\mathrm{cm}^{-1}\right)$ : 1714 (OAc), 1680 (NAc), $1611(\mathrm{C}=\mathrm{N})$; MS (70 eV) $m / z(\%): 452\left(\mathrm{M}^{+}, 7\right)$. Anal. calcd for $\mathrm{C}_{24} \mathrm{H}_{28} \mathrm{~N}_{4} \mathrm{O}_{5}: \mathrm{C}, 63.70 ; \mathrm{H}$, 6.24; N, 12.38; found C, 63.68; H, 6.28; N, 12.37 .

General procedure for the synthesis of 1-(alditol-1-yl)1,2,4-triazolo[4,3-a]phthalazines (22 a-e): A 2M solution of iron (III) chloride in absolute ethanol $(2 \mathrm{~mL})$ was added dropwise to a boiling solution of $\mathbf{2 0 a}-\mathbf{e}(0.01 \mathrm{~mol})$ in ethanol $(50 \mathrm{~mL})$. Heating was continued for $20 \mathrm{~min}$ and the mixture was then kept overnight at room temperature. The product was filtered, washed repeatedly with water, dried and recrystallized from ethanol to give compounds 22a-e.

4-(2,4,6-Trimethylphenyl)-1-(D-gluco-pentitol-1-yl)1,2,4-triazolo[4,3-a]phthalazine (22a): Yield: $89 \%$; m.p. 86$87{ }^{\circ} \mathrm{C} ;{ }^{1} \mathrm{H}$ NMR (DMSO- $\left.d_{6}\right) \delta: 2.34\left(\mathrm{~s}, 3 \mathrm{H}, \mathrm{CH}_{3}\right), 2.50(\mathrm{~s}, 6 \mathrm{H}$, $2 \mathrm{CH}_{3}$ ), 3.33-3.62 (protons of the alditol congregated with the solvent absorption), 3.73-3.90 (m, 2H, $\left.\mathrm{CH}_{2} \mathrm{OH}\right), 4.31-5.22(\mathrm{~m}$, 5H, 5OH, $\mathrm{D}_{2} \mathrm{O}$ exchangeable), 7.09-8.09 (m, 6H, Ar- $\left.\mathrm{H}\right) ;{ }^{13} \mathrm{C}$ NMR (DMSO- $d_{6}$ ) $\delta: ~ 20.5,22.1,63.4,66.3,70,71.8,73.5$, 122.2, 126.7, 126.9, 127.7, 129, 130.8, 131.5, 132, 137.3, 138, 149.7, 154, 160.1; IR (KBr, $\left.v_{\max }, \mathrm{cm}^{-1}\right): 3454-3219(\mathrm{OH}), 1605$ $(\mathrm{C}=\mathrm{N})$; MS $(70 \mathrm{eV}) \mathrm{m} / \mathrm{z}(\%): 438\left(\mathrm{M}^{+}, 9\right)$. Anal. calcd for $\mathrm{C}_{23} \mathrm{H}_{26} \mathrm{~N}_{4} \mathrm{O}_{5}$ : C, 63; H, 5.98; N, 12.78; found.C, 63.05; H, 5.96; $\mathrm{N}, 12.80$.

4-(2,4,6-Trimethylphenyl)-1-(D-galacto-pentitol-1-yl)1,2,4-triazolo[4,3-a]phthalazine (22b): Yield: $85 \%$; m.p. 81$82{ }^{\circ} \mathrm{C} ;{ }^{1} \mathrm{H}$ NMR (DMSO- $\left.d_{6}\right) \delta: 2.31\left(\mathrm{~s}, 3 \mathrm{H}, \mathrm{CH}_{3}\right), 2.51(\mathrm{~s}, 6 \mathrm{H}$, $2 \mathrm{CH}_{3}$ ), 3.30-3.61 (protons of the alditol congregated with the solvent absorption), 3.74-3.92 (m, 2H, $\left.\mathrm{CH}_{2} \mathrm{OH}\right), 4.31-5.32$ (m, $5 \mathrm{H}, 5 \mathrm{OH}, \mathrm{D}_{2} \mathrm{O}$ exchangeable), 7.01-8.10 (m, 6H, Ar-H); ${ }^{13} \mathrm{C}$ NMR (DMSO- $\left.d_{6}\right) \delta: 20.6,22.1,63.8,65.2,70.1,70.8,72.2$, $122.5,126,126.9,128.9,129.7,130.8,131.7,132.2,136.4$, 138.1, 148.4, 154.2, 160.6; IR (KBr, $\left.v_{\max }, \mathrm{cm}^{-1}\right)$ : 3459-3279 $(\mathrm{OH}), 1606(\mathrm{C}=\mathrm{N})$. Anal. calcd. for $\mathrm{C}_{23} \mathrm{H}_{26} \mathrm{~N}_{4} \mathrm{O}_{5}: \mathrm{C}, 63 ; \mathrm{H}$, $5.98 ; \mathrm{N}, 12.78$; found $\mathrm{C}, 63.03 ; \mathrm{H}, 5.99 ; \mathrm{N}, 12.82$.

4-(2,4,6-Trimethylphenyl)-1-(D-manno-pentitol-1-yl)1,2,4-triazolo[4,3-a]phthalazine (22c): Yield: $73 \%$; m.p. 106$107{ }^{\circ} \mathrm{C} ;{ }^{1} \mathrm{H}$ NMR (DMSO- $\left.d_{6}\right) \delta: 2.39\left(\mathrm{~s}, 3 \mathrm{H}, \mathrm{CH}_{3}\right), 2.50(\mathrm{~s}$, $6 \mathrm{H}, 2 \mathrm{CH}_{3}$ ), 3.22-3.60 (protons of the alditol congregated with the solvent absorption), 3.70-3.86 ( $\left.\mathrm{m}, 2 \mathrm{H}, \mathrm{CH}_{2} \mathrm{OH}\right), 4.30-5.36$ (m, 5H, 5OH, $\mathrm{D}_{2} \mathrm{O}$ exchangeable), 7.02-8.13 (m, 6H, Ar-H); ${ }^{13} \mathrm{C}$ NMR (DMSO- $\left.d_{6}\right) \delta: 20.1,23.2,63.0,66.7,70.3,71.8$, 72.5, 123.5, 126.1, 126.8, 128.0, 129.3, 130.4, 131.7, 132.5, $137.4,138,146.4,154,161.3$; IR (KBr, $\left.v_{\max }, \mathrm{cm}^{-1}\right): 3478-3210$
(OH), $1602(\mathrm{C}=\mathrm{N}) ; \mathrm{MS}(70 \mathrm{eV}) \mathrm{m} / z(\%): 438\left(\mathrm{M}^{+}, 6\right)$. Anal. calcd. for $\mathrm{C}_{23} \mathrm{H}_{26} \mathrm{~N}_{4} \mathrm{O}_{5}$ : C, 63; H, 5.98; N, 12.78; found C, 63.09; H, 5.90; N, 12.70.

4-(2,4,6-Trimethylphenyl)-1-(D-xylo-pentitol-1-yl)1,2,4-triazolo[4,3-a]phthalazine (22d): Yield: $60 \%$; m.p. 125-126 ${ }^{\circ} \mathrm{C}$; ${ }^{1} \mathrm{H}$ NMR (DMSO- $d_{6}$ ) $\delta: 2.30$ (s, $3 \mathrm{H}, \mathrm{CH}_{3}$ ), 2.48 (s, $\left.6 \mathrm{H}, 2 \mathrm{CH}_{3}\right), 3.20-3.60$ (the protons of the alditol congregated with the solvent absorption), 3.65-3.77 (m, $\left.2 \mathrm{H}, \mathrm{CH}_{2} \mathrm{OH}\right), 3.91-$ $5.14\left(\mathrm{~m}, 4 \mathrm{H}, 4 \mathrm{OH}, \mathrm{D}_{2} \mathrm{O}\right.$ exchangeable), 7-8.03 (m, 6H, Ar$\mathrm{H}) ;{ }^{13} \mathrm{C}$ NMR (DMSO- $d_{6}$ ) $\delta: 20.2,22.8,63.0,66.1,73.8,74.5$, 124, 126.3, 127.1, 128.5, 129.6, 130.8, 131.4, 132.3, 136.6, 138.1, 147.2, 154.0, 160.1; IR (KBr, $\left.v_{\max }, \mathrm{cm}^{-1}\right)$ : 3455-3266 $(\mathrm{OH}), 1607(\mathrm{C}=\mathrm{N})$. Anal. calcd. for $\mathrm{C}_{22} \mathrm{H}_{24} \mathrm{~N}_{4} \mathrm{O}_{4}: \mathrm{C}, 64.69 ; \mathrm{H}$, 5.92; N, 13.72; found C, C, 64.67; H, 5.90; N, 13.76 .

4-(2,4,6-Trimethylphenyl)-1-(D-arabino-pentitol-1-yl)1,2,4-triazolo[4,3-a]phthalazine (22e): Yield: $63 \%$; m.p. 92$93{ }^{\circ} \mathrm{C} ;{ }^{1} \mathrm{H}$ NMR (DMSO- $d_{6}$ ) $\delta: 2.33$ (s, 3H, $\mathrm{CH}_{3}$ ), 2.49 (s, 6H, $2 \mathrm{CH}_{3}$ ), 3.16-3.54 (the protons of the alditol congregated with the solvent absorption), 3.60-3.79 (m, $\left.2 \mathrm{H}, \mathrm{CH}_{2} \mathrm{OH}\right), 3.90-5.24$ (m, 4H, 4OH, $\mathrm{D}_{2} \mathrm{O}$ exchangeable), 7.11-8 (m, 6H, Ar-H); ${ }^{13} \mathrm{C}$ NMR (DMSO- $\left.d_{6}\right) \delta: 21,23.5,64.3,66.7,72.9,74.4,124.1$, 127.3, 127.6, 128.5, 129.5, 130.4, 131.7, 132, 137.6, 139.2, 147, 154.2, 161.2; IR (KBr, $\left.v_{\max }, \mathrm{cm}^{-1}\right): 3472-3196(\mathrm{OH}), 1604$ $(\mathrm{C}=\mathrm{N})$; MS $(70 \mathrm{eV}) \mathrm{m} / \mathrm{z}(\%): 408\left(\mathrm{M}^{+}, 11\right)$. Anal. calcd. for $\mathrm{C}_{22} \mathrm{H}_{24} \mathrm{~N}_{4} \mathrm{O}_{4}$ : C, 64.69; H, 5.92; N, 13.72; found C, C, 64.60; H, 5.91; N, 13.70.

General procedure for the synthesis of (penta- $O$-acetylsugar-1-yl)-1,2,4-triazolo[4,3-a]phthalazines (23 a-e): A cold solution of 22 a-e $(0.01 \mathrm{~mol})$ in dry pyridine $(10 \mathrm{~mL})$ was treated with acetic anhydride $(10 \mathrm{~mL})$ and the mixture was kept overnight at room temperature with occasional shaking. The mixture was poured onto crushed ice and the product was collected by filtration, washed repeatedly with water, dried and recrystallized from ethanol to give compounds 23 a-e.

4-(2,4,6-Trimethylphenyl)-1-(1,2,3,4,5-penta- $O$-acetylD-gluco-pentitol-1-yl)-1,2,4-triazolo[4,3-a]phthalazine (23a): Yield: $75 \%$; m.p. $70-71{ }^{\circ} \mathrm{C}$; ${ }^{1} \mathrm{H}$ NMR (DMSO- $d_{6}$ ) $\delta$ : 1.99, 2.01, 2.03 (3s, 15H, 5OAc), 2.39 (s, 3H, $\left.\mathrm{CH}_{3}\right), 2.50$ (s, $\left.6 \mathrm{H}, 2 \mathrm{CH}_{3}\right), 3.98-4.01$ (m, $\left.2 \mathrm{H}, \mathrm{CH}_{2} \mathrm{OAc}\right), 4.23-5.59$ (m, 4H, 4CHOAc), 7.03-8.10 (m, 6H, Ar-H); $\left.{ }^{13} \mathrm{C} \mathrm{NMR} \mathrm{(DMSO-} d_{6}\right) \delta$ : 19, 19.8, 21.5, 22.6, 62.1, 67.1, 69.2, 70.6, 77.4, 125.0, 127.3, $128,128.5,129.8,130.8,131.9,133,137.6,140.2,148,154.2$, 160.2, 174.2; IR (KBr, $\left.v_{\max }, \mathrm{cm}^{-1}\right)$ : $1724(\mathrm{OAc}), 1601(\mathrm{C}=\mathrm{N})$. Anal. calcd. for $\mathrm{C}_{33} \mathrm{H}_{36} \mathrm{~N}_{4} \mathrm{O}_{10}$ : C, 61.10; H, 5.59; N, 8.64; found C, 61.11; H, 5.58; N, 8.60.

4-(2,4,6-Trimethylphenyl)-1-(1,2,3,4,5-penta-O-acetylD-galacto-pentitol-1-yl)-1,2,4-triazolo[4,3-a]phthalazine (23b). Yield: $80 \%$; m.p. $63-64{ }^{\circ} \mathrm{C}$; ${ }^{1} \mathrm{H}$ NMR (DMSO- $\left.d_{6}\right) \delta$ : 1.98-2.25 (m, 15H, $5 \mathrm{O} \mathrm{Ac}), 2.38\left(\mathrm{~s}, 3 \mathrm{H}, \mathrm{CH}_{3}\right), 2.51(\mathrm{~s}, 6 \mathrm{H}$, $\left.2 \mathrm{CH}_{3}\right)$, 3.94-4.03 (m, 2H, $\left.\mathrm{CH}_{2} \mathrm{OAc}\right), 4.21-5.55(\mathrm{~m}, 4 \mathrm{H}$, 4CHOAc), 7-8.11 (m, 6H, Ar-H); ${ }^{13} \mathrm{C}$ NMR (DMSO- $\left.d_{6}\right) \delta$ : 19.03, 20, 21.56, 23, 62.2, 68.1, 69.1, 70.6, 76. 5, 123, 1276.3, $128,128.6,129.8,130.2,132.3,133.1,137.1,141.2,147$, 154.2, 160, 174.5; IR (KBr, $v_{\max }, \mathrm{cm}^{-1}$ ): 1730 (OAc), 1606 $(\mathrm{C}=\mathrm{N})$; MS (70 eV) $\mathrm{m} / \mathrm{z}(\%): 648\left(\mathrm{M}^{+}, 6\right)$. Anal. calcd. for $\mathrm{C}_{33} \mathrm{H}_{36} \mathrm{~N}_{4} \mathrm{O}_{10}$ : C, 61.10; H, 5.59; N, 8.64; found.C, 61.17; H, $5.55 ; \mathrm{N}, 8.64$.

4-(2,4,6-Trimethylphenyl)-1-(1,2,3,4,5-penta- $O$-acetylD-manno-pentitol-1-yl)-1,2,4-triazolo[4,3-a]phthalazine 
(23c). Yield: $60 \%$; m.p. $71-72{ }^{\circ} \mathrm{C}$; ${ }^{1} \mathrm{H}$ NMR (DMSO- $\left.d_{6}\right) \delta$ : 1.96-2.28 (m, 15H, 5OAc), $2.33\left(\mathrm{~s}, 3 \mathrm{H}, \mathrm{CH}_{3}\right), 2.48(\mathrm{~s}, 6 \mathrm{H}$, $2 \mathrm{CH}_{3}$ ), 3.90-4 (m, $\left.2 \mathrm{H}, \mathrm{CH}_{2} \mathrm{OAc}\right), 4.20-5.57$ (m, 4H, 4CHOAc), 7.01-8.13(m, 6H, Ar-H); ${ }^{13} \mathrm{C}$ NMR (DMSO- $\left.d_{6}\right) \delta: 18.9,19.7$, 21.2, 22.9, 63, 67.2, 69.2, 70.6, 77.7, 126, 127.1, 128.2, 128.7, 129.6, 130.8, 131.8, 133, 137, 141.2, 147.6, 153.1, 159.2, 173.8; IR (KBr, $\left.v_{\max }, \mathrm{cm}^{-1}\right)$ : $1735(\mathrm{OAc}), 1601(\mathrm{C}=\mathrm{N})$. Anal. calcd. for $\mathrm{C}_{33} \mathrm{H}_{36} \mathrm{~N}_{4} \mathrm{O}_{10}$ : C, 61.10; H, 5.59; N, 8.64; found.C, $61.14 ; \mathrm{H}, 5.50 ; \mathrm{N}, 8.60$.

4-(2,4,6-Trimethylphenyl)-1-(1,2,3,4,5-penta- $O$-acetylD-xylo-pentitol-1-yl)-1,2,4-triazolo[4,3-a]phthalazine (23d). Yield: $55 \%$; m.p. $68-69{ }^{\circ} \mathrm{C}$; ${ }^{1} \mathrm{H}$ NMR (DMSO- $\left.d_{6}\right) \delta$ : 1.86-2.17 (m, 12H, 4OAc), 2.36 (s, 3H, $\left.\mathrm{CH}_{3}\right), 2.49$ (s, 6H, $2 \mathrm{CH}_{3}$ ), 3.94-4 (m, 2H, $\mathrm{CH}_{2} \mathrm{OAc}$ ), 4.23-5.22 (m, 3H, 3CHOAc), 7.11-8.14 (m, 6H, Ar-H); ${ }^{13} \mathrm{C}$ NMR (DMSO- $d_{6}$ ) $\delta: 19.1,20$, 21.4, 23.8, 63.2, 68.1, 69.4, 75.8, 125.6, 127.2, 127.9, 128.7, 129.1, 130, 131.3, 132.9, 137.3, 141.4, 148.6, 152.2, 157.7, 172.9; IR (KBr, $\left.v_{\max }, \mathrm{cm}^{-1}\right)$ : 1721 (OAc), $1607(\mathrm{C}=\mathrm{N})$; $\mathrm{MS}(70$ eV) $m / z(\%): 576\left(\mathrm{M}^{+}, 9\right)$. Anal. calcd. for $\mathrm{C}_{30} \mathrm{H}_{32} \mathrm{~N}_{4} \mathrm{O}_{8}: \mathrm{C}, 62.49$; H, 5.59; N, 9.72; found.C, 62.40; H, 5.62; N, 9.70 .

4-(2,4,6-Trimethylphenyl)-1-(1,2,3,4,5-penta- $O$-acetylD-arabino-pentitol-1-yl)-1,2,4-triazolo[4,3-a]phthalazine (23e). Yield: $65 \%$; m.p. 93-94 ${ }^{\circ} \mathrm{C}$; ${ }^{1} \mathrm{H}$ NMR (DMSO- $d_{6}$ ) $\delta$ : 1.89-2.14 (m, 12H, 4OAc), 2.31 (s, 3H, $\left.\mathrm{CH}_{3}\right), 2.47$ (s, 6H, $\left.2 \mathrm{CH}_{3}\right), 3.90-3.99\left(\mathrm{~m}, 2 \mathrm{H}, \mathrm{CH}_{2} \mathrm{OAc}\right), 4.21-5.20(\mathrm{~m}, 3 \mathrm{H}$, 3CHOAc), 7.10-8.10 (m, 6H, Ar-H); $\left.{ }^{13} \mathrm{C} \mathrm{NMR} \mathrm{(DMSO-} d_{6}\right) \delta$ : 19.2, 20.01, 21, 22.8, 64.1, 68, 69, 74.1, 125.7, 127, 127.9, 128.7, 129, 130.3, 131.6, 132.5, 138.3, 141, 148.1, 152, 157, 175.2; IR (KBr, $\left.v_{\max }, \mathrm{cm}^{-1}\right)$ : $1733(\mathrm{OAc}), 1604(\mathrm{C}=\mathrm{N})$. Anal. calcd. for $\mathrm{C}_{30} \mathrm{H}_{32} \mathrm{~N}_{4} \mathrm{O}_{8}$ : C, 62.49; H, 5.59; N, 9.72; found $\mathrm{C}$, 62.44; H, 5.60; N, 9.71 .

\section{RESULTS AND DISCUSSION}

The key precursor 1-chloro-4-(2,4,6-trimethylphenyl) phthalazine $\mathbf{2}$ was prepared in moderate yield upon refluxing of phthalazinone derivative $\mathbf{1}$ and a mixture of phosphorous oxychloride/phosphorous pentachloride on a water bath, which upon subsequent reaction with hydrazine hydrate in refluxing ethanol afforded the target compound, hydrazinophthalazine derivative 3 (Scheme-I) ${ }^{51}$.<smiles>O=c1[nH]nc(Br)c2ccccc12</smiles>

1<smiles>Cc1cc(C)c(C)c(C)c1</smiles>

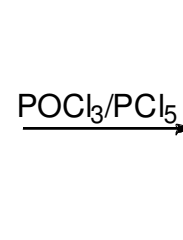<smiles>Clc1nnc(Br)c2ccccc12</smiles>

2

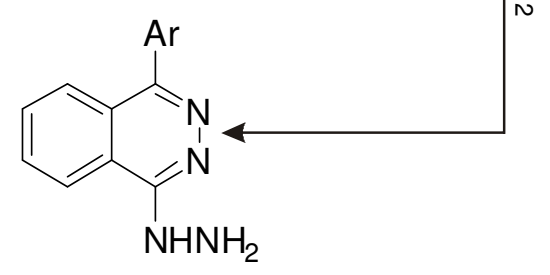

3
The chemical behavior of chlorophthalazine derivative 2 towards active methylene compounds was studied with respect to the synthesis of highly substituted pyrazoles ${ }^{52,53}$. Thus, the treatment of compound $\mathbf{2}$ with different active methylene compounds namely,malononitrile, ethyl cyanoacetate, ethyl acetoacetate and acetylacetone afforded the corresponding phthalazine derivatives 4-6, respectively. The IR spectrum of compound $\mathbf{4}$ and $\mathbf{5 a}$ revealed the presence of a $\mathrm{CN}$ group at 2223 and $2231 \mathrm{~cm}^{-1}$, respectively. The ${ }^{1} \mathrm{H}$ NMR spectrum of compounds 4-6 displayed a $\mathrm{CH}$ proton as a singlet signalat $\delta$ 4.57-5.11 ppm. The ${ }^{13} \mathrm{C}$ NMR spectrum of compound 6 gave characteristic signals in accordance with the assigned structure. Cyclocondensation of compounds 4-6 with hydrazine hydrate in refluxing absolute ethanol yielded the pyrazolylphthalazine derivatives 7-9, respectively. The infrared spectrum of compound 7 showed the absence of nitrile functional group and the presence of only $\mathrm{NH}_{2}$ groups (Scheme-II).

In this study, our attention was also directed towards the synthesis of pyrazolylphthalazine derivatives in order to attain possible potential antihypertensive agents ${ }^{54}$. Thus, hydrazinophthalazine derivative $\mathbf{3}$ was subjected to react with different active methylene compounds namely, ethyl acetoacetate, ethyl cyanoacetate, diethyl malonate and acetylacetone to afford the corresponding pyrazolylphthalazine derivatives ${ }^{10-13}$, respectively. The IR spectra of compounds ${ }^{10-12}$ revealed the presence of a CO group 1707-1666 $\mathrm{cm}^{-1}$ region. The ${ }^{1} \mathrm{H}$ NMR spectrum of $\mathbf{1 1}$ showed a signal at 4.47 for assigned $\mathrm{NH}_{2}$ (exchangeable with $\mathrm{D}_{2} \mathrm{O}$ ). The mass spectrum of $\mathbf{1 3}$ showed a peak corresponding to its molecular ion at $m / z$ 342. Moreover, treatment of 3 with acrylonitrile in refluxing pyridine afforded the corresponding propionitrile derivative $\mathbf{1 4}$, which underwent acid hydrolysis to give pyrazolylphthalazine derivative 15 (SchemeIII). The IR spectrum of compound $\mathbf{1 4}$ showed the presence of an absorption band for $\mathrm{CN}$ group at $2221 \mathrm{~cm}^{-1}$. The mass spectrum of $\mathbf{1 5}$ showed a peak corresponding to its molecular ion at $m / z 331$.

Similarly, the hydrazinophthalazine derivative $\mathbf{3}$ has been reacted with ethoxymethylenemalononitrile, tetracyanoethylene, bis(methylthio)-methylenemalononitrile, or ethyl(ethoxymethylene)-cyanoacetate in refluxing ethanol to produce the corresponding pyrazole derivatives 16-19, respectively. The structures of the latter compounds were confirmed on the basis of their elemental analysis and spectral data. The IR spectrum of compounds 16 and 17 showed absorption bands characteristic for $\mathrm{NH}_{2}$ and $\mathrm{C} \equiv \mathrm{N}$ groups, while those of compounds 18 and 19 revealed absorption bands characteristic for $\mathrm{NH}_{2}$ and $\mathrm{C}=\mathrm{O}$. Moreover, the ${ }^{1} \mathrm{H}$ NMR spectrum of compounds 16-19 showed signals at $\delta=6.88,6.92,6.94$ and $6.90 \mathrm{ppm}$ for assigned $\mathrm{NH}_{2}$ (exchangeable with $\mathrm{D}_{2} \mathrm{O}$ ), respectively. The mass spectrum of compounds 16-19 gave the molecular ion peaks at $m / z=354,379,401$ and 447, respectively (Scheme-IV).

The scope of this examination was further prolonged towards the synthesis of acyclo- $C$-nucleoside derivatives. Thus, condensation of hydrazinophthalazine derivative $\mathbf{3}$ with equimolar amount of different aldohexose and aldopentose namely, D-glucose, D-galactose, D-mannose, D-xylose and $\mathrm{D}$-arabinose, respectively in refluxing ethanol in presence of catalytic amount of acetic acid gave the corresponding sugar 


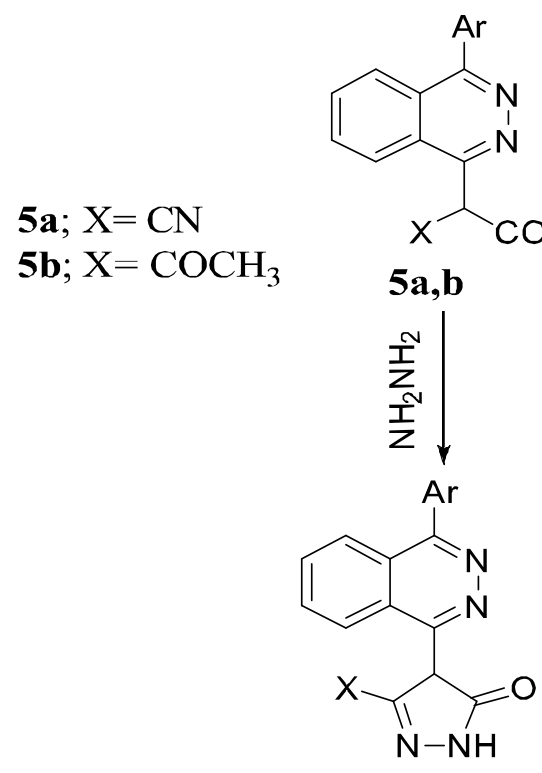

$\mathbf{8 a}, \mathbf{b}$

8a; $X=\mathrm{NH}_{2}$

$\mathbf{8 b} ; \mathrm{X}=\mathrm{CH}_{3}$<smiles>Clc1nnc(Br)c2ccccc12</smiles><smiles>C1O[C@@H]2C[C@@H]1O2</smiles><smiles>CC(=O)C(C(C)=O)c1nnc([Al])c2ccccc12</smiles>

6<smiles>N#CC(C#N)c1nnc(Br)c2ccccc12</smiles>

4

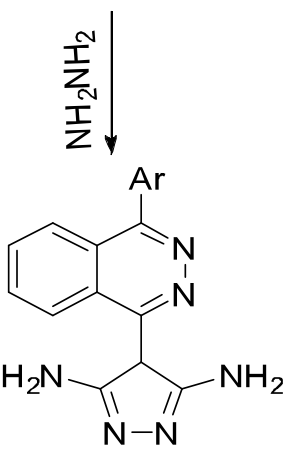

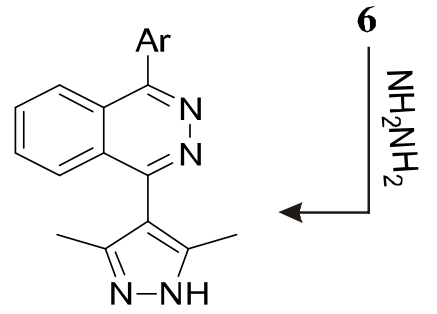

\section{7}

9

Scheme-II: Synthesis of pyrazolylphthalazine derivatives 4-9<smiles>Cc1cc(C)n(-c2nnc(Br)c3ccccc23)n1</smiles><smiles>[H][Y]([H])(CC)c1nnc(N2NC(=O)CC2=O)c2ccccc12</smiles><smiles>CCCCCCC(=O)OCC</smiles><smiles>NNc1nnc(Br)c2ccccc12</smiles>

$\mathrm{CH}_{3} \mathrm{COCH}_{2} \mathrm{COOEt}$

3<smiles>CC1=NN(c2nnc(Br)c3ccccc23)C(=O)C1</smiles>

$\mathrm{CH}_{2}(\mathrm{COOEt})_{2}$

$\mathrm{CH}_{2}=\mathrm{CHCN}$

10<smiles></smiles><smiles>Cc1nnc([Al][Te])c2ccccc12</smiles>

$14 \mathrm{NHNHCH}_{2} \mathrm{CH}_{2} \mathrm{CN}$ 


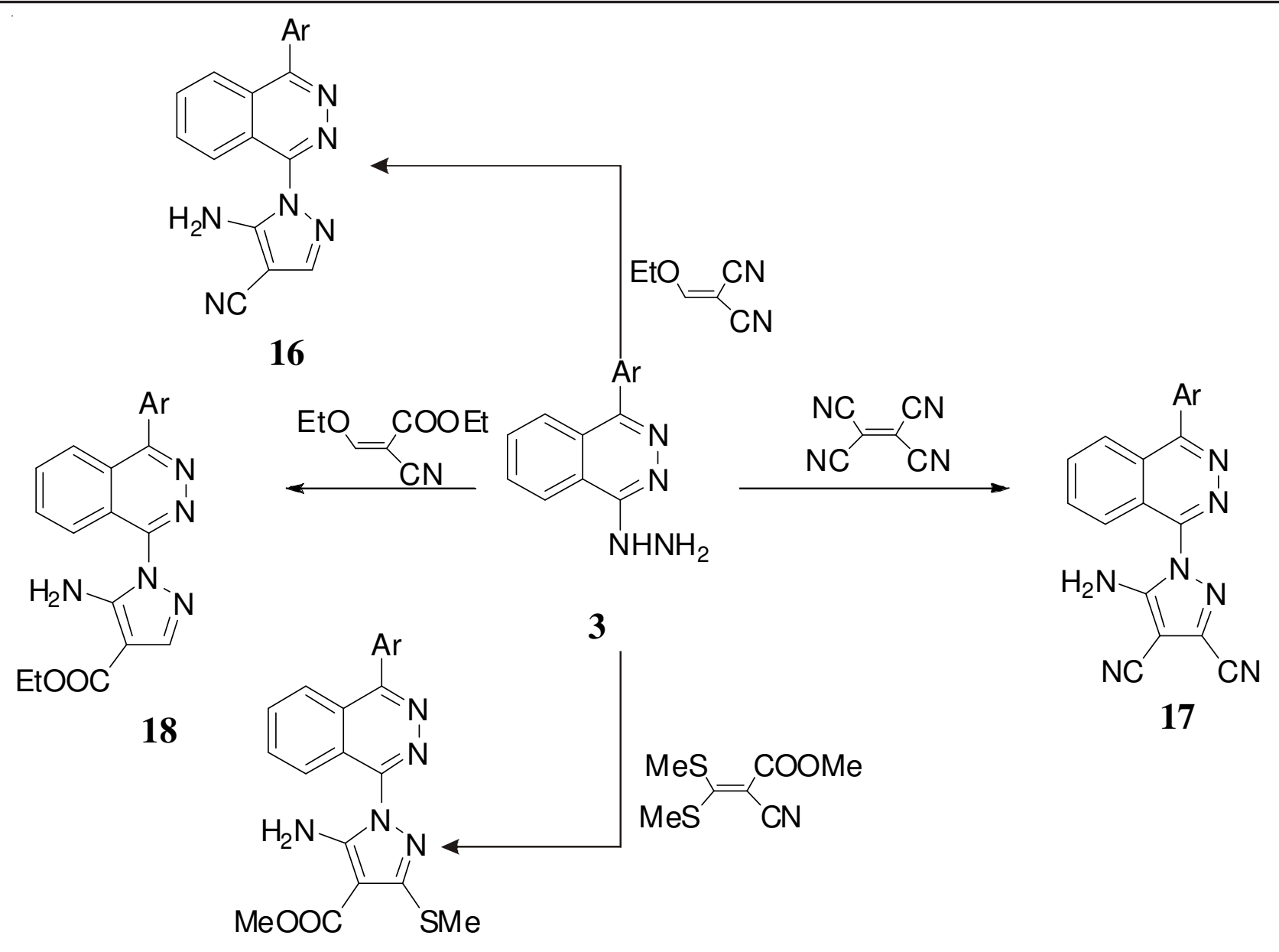

19

Scheme-IV: Synthesis of pyrazolylphthalazine derivatives 17-19

hydrazones 20 a-e. The IR spectrum of compounds 20 a-e showed characteristic absorption bands $3438-3205 \mathrm{~cm}^{-1}$ region corresponding to the $\mathrm{OH}$ and $\mathrm{NH}$ groups. Acetylation of the sugar hydrazones $\mathbf{2 0}$ a-e with acetic anhydride in dry pyridine at room temperature afforded the corresponding per-acetyl derivatives 21 a-e, whose IR spectra showed the disappearance of the $\mathrm{OH}$ groups and appearance of absorption bands in the carbonyl frequency region at 1726-1713 and 1685-1674 $\mathrm{cm}^{-1}$ due to (OAc) and (NAc) groups, respectively. Their ${ }^{1} \mathrm{H}$ NMR spectra showed signals corresponding to $O$-acetyl groups in addition to NAc groups; whereas no signals could be found for $\mathrm{NH}$ groups confirming that per- $\mathrm{O}$ - and $\mathrm{N}$-acetylation had taken place. The spectra also showed the presence of the $\mathrm{CH}=\mathrm{N}$ proton as doublet at low field at $\delta 6.55-6.74$ ppmin addition to the rest of the alditol-1-yl side chain. The sugar hydrazone 20 a-e on oxidative cyclization with iron(III) chloride in ethanolic solution afforded the corresponding triazolo[4,3a]phthalazine derivatives 22a-e. This oxidation may take place by the electrophilic attack of the hard acid site of iron(III) chloride ${ }^{55}$ on the hardest basic site of the sugar hydrazone 20 a-e, followed by the elimination of hydrogen chloride and formation of possibly a nitrilimine that undergoes 1,5 -electrocyclization to give $\mathbf{2 2}$ a-e. Their IR spectra showed characteristic absorption bands at $3478-3196 \mathrm{~cm}^{-1}$ corresponding to the $\mathrm{OH}$ groups. The mass spectrum of 22a showed a peak correspon- ding to its molecular ion at $m / z 438$, in addition to ion peak at $\mathrm{m} / \mathrm{z} 287$ presumably attributed to the triazolophthalazine ring. The ${ }^{1} \mathrm{H}$ NMR spectrum of compound $\mathbf{2 2} \mathbf{b}$ showed a doublet at low field at $\delta 3.90 \mathrm{ppm}$ which was assigned to $\mathrm{H}-1$, followed by the rest of the aldito-1-yl side chain at higher field. The spectra of 22 c-e showed a similar pattern. Acetylation of 22ae with acetic anhydride in pyridine afforded the polyacetoxyalkyl derivatives 23a-e (Scheme-V), whose IR spectra showed the presence of only one absorption band in the carbonyl frequency region (OAc) and devoid any band for $\mathrm{OH}$. The ${ }^{1} \mathrm{H}$ NMR spectra confirmed the presence of the OAc groups. The mass spectrum of 23b and 23d showed a peak corresponding to its molecular ion at $m / z 648$ and 576, which when combined with the elemental analysis led to the assignment of the molecular formula $\mathrm{C}_{33} \mathrm{H}_{36} \mathrm{~N}_{4} \mathrm{O}_{10}$ and $\mathrm{C}_{30} \mathrm{H}_{32} \mathrm{~N}_{4} \mathrm{O}_{8}$, respectively.

\section{Biological activities}

Antibacterial: Antimicrobial activity of the newly synthesized compounds 7-13, 15-19, 22 a-e and 23a-e were assessed against Escherichia coli, Staphylococcus aureus, Bacillus subtilis, Salmonella typhi bacterial strains, Aspergillumsniger and Candida albicans fungal strains in term of disk diffusion method ${ }^{62}$. As described by El-Hashash et al. ${ }^{62}$, amoxicillin and ketoconazole were used as a standard drugs for the bacteria and fungi, respectively. Preliminary screening of phthalazine-derivatives 


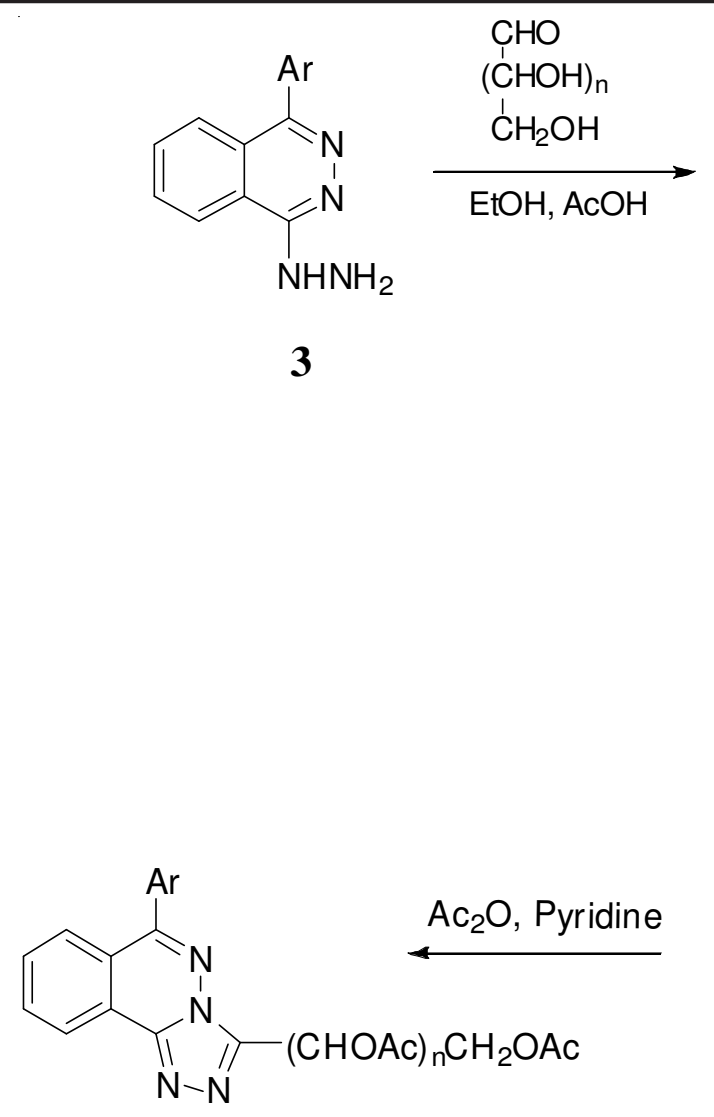

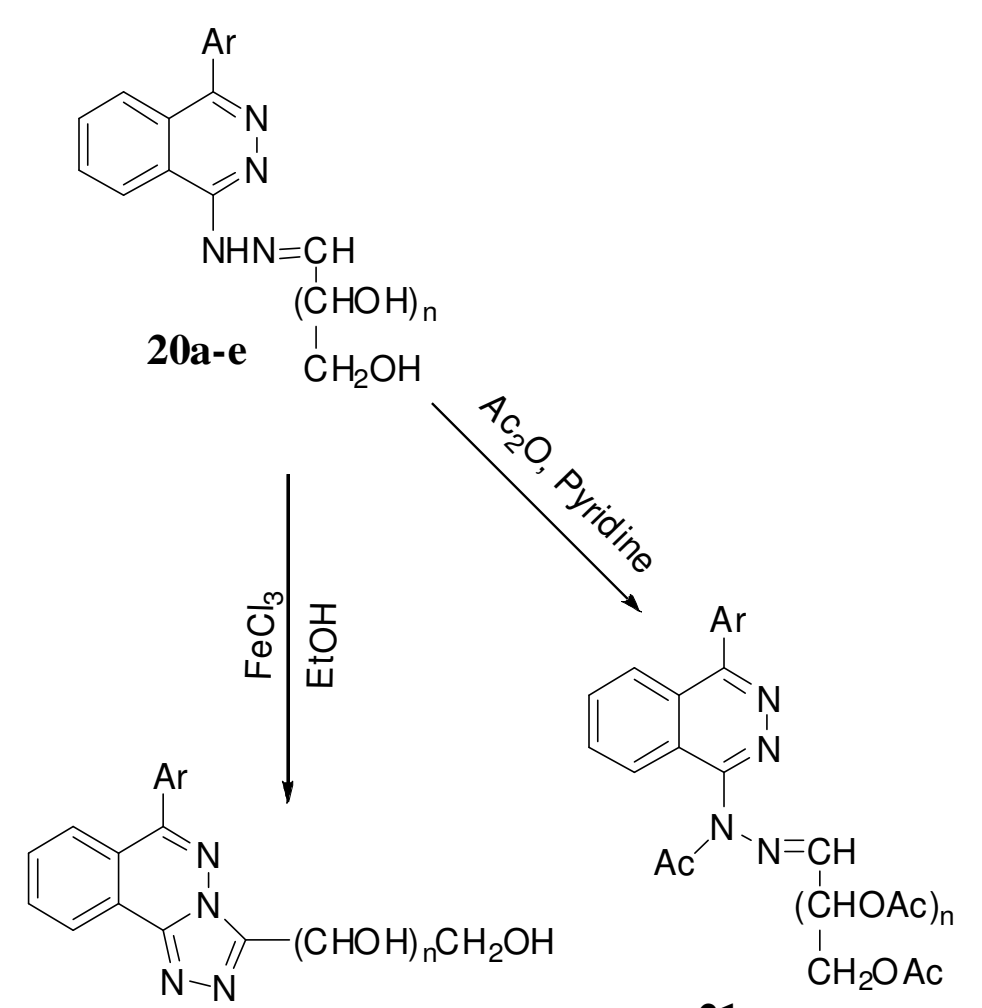

21a-e

23a-e<smiles>CC(C)OC(C)C(C)O</smiles>

a<smiles>CC(O)C(O)C(O)C(C)CO</smiles>

b<smiles>CC(O)C(O)(O)C(C)(O)CO</smiles>

c<smiles>CC(O)C(O)C(C)O</smiles>

d e

Scheme-V: Synthesis of acyclo- $C$-nucleosides of 6-(2,4,6-trimethylphenyl)-1,2,4-triazolo[3,4-a]phthalazine 22 and 23 a-e

and standard drugs were accomplished at fixed concentrations $(500 \mu \mathrm{g} / \mathrm{mL})$. The antimicrobial activity was noted after $24 \mathrm{~h}$, by calculating the inhibition zone diameter for bacteria and after $72 \mathrm{~h}$ in case of fungi. Each test was repeated twice. Based on the results of inhibition zone, the minimum inhibitory concentration (MIC) of compounds 7-13, 15-19, 22 a-e and 23 a-e against all strains; bacterial and fungal was determined by liquid dilution method ${ }^{62}$. As described by El-Hashash et al. ${ }^{62}$ stock solutions of the tested compounds with 500, 250, 200, 100, $62.5,50,25$ and $12.5 \mu \mathrm{g} \mathrm{mL}^{-1}$ concentrations were dilutedusing DMSO as solvent. The same concentrations of standard drugs e.g., amoxicillin and ketoconazole, were also prepared. Inoculums of both bacterial and fungal culture were also prepared. To a series of test tubes containing $1 \mathrm{~mL}$ each of phthalazine compound solution with different concentrations and $0.2 \mathrm{~mL}$ of the inoculums was added. Further $3.8 \mathrm{~mL}$ of sterile water was added to each of the test tubes. These tubes were incubated for $24 \mathrm{~h}$ at $37^{\circ} \mathrm{C}$ and the turbidity for all tubes was observed. This method was repeated by changing phthalazine compounds with standard drugs e.g., amoxicillin and ketoconazole for comparison. The minimum inhibitory concentration at which no growth was detected (Table-1). The comparison of the MICs (in $\mu \mathrm{g} / \mathrm{mL}$ ) of potent compounds and standard drugs against tested strains are presented in the Table-1.

Antiinflammatory activity: A series of phthalazin-4-yl acetic acid derivatives ${ }^{56}$ was prepared and showed antiinflammatory activity in the carrageenin and edema test. Recently, several groups have studied the structure-activity relationship of novel series of 4-arylsubstituted phthalazinones ${ }^{28,57}$ and showed that the presence of 4-aryl substituted on the phthalazinone nucleus contributes to a good antiinflammatory and nociceptive activities. It is also well known that some heteroaryl acetic acids possess antiinflammatory activities ${ }^{58,59}$. Substitution of the acetamide side chain on the lactam nitrogen in the phthalazinone derivatives ${ }^{25}$ resulted in compounds having considerably high antinociceptive and antiinflammatory activities without gastric lesion and bleeding at the given dose. It was of interest to prepare a number of 4-aryl-oxophthalazine- 


\begin{tabular}{|c|c|c|c|c|c|c|}
\hline \multirow{4}{*}{ Compounds } & \multicolumn{6}{|c|}{$\begin{array}{l}\text { TABLE-1 } \\
\text { ANTIMICROBIAL ACTIVITY OF } \\
\text { MPOUNDS 7-13, 15-19, 22a-e AND 23a-e }\end{array}$} \\
\hline & \multicolumn{6}{|c|}{ Minimum inhibitory concentration (MIC) in $\mu \mathrm{g} / \mathrm{mL}$} \\
\hline & \multicolumn{3}{|c|}{ Bacterial strains } & \multicolumn{3}{|c|}{ Fungal strains } \\
\hline & $\begin{array}{c}S . \\
\text { aureus }\end{array}$ & $\begin{array}{c}B . \\
\text { subtilis }\end{array}$ & $\begin{array}{c}S . \\
\text { typhi }\end{array}$ & $\begin{array}{c}E . \\
\text { coli } \\
\end{array}$ & $\begin{array}{c}A . \\
\text { niger }\end{array}$ & $\begin{array}{c}C . \\
\text { albican }\end{array}$ \\
\hline 7 & 50 & 25 & 25 & 50 & 125 & 125 \\
\hline 8a & 50 & 50 & 25 & 25 & 250 & 125 \\
\hline $\mathbf{8 b}$ & 50 & 100 & 200 & 200 & 250 & - \\
\hline 9 & 500 & 250 & - & 500 & 500 & - \\
\hline 10 & 250 & 500 & 200 & 200 & - & 500 \\
\hline 11 & 50 & 25 & 25 & 50 & 62.5 & 125 \\
\hline 12 & 100 & 100 & 250 & 100 & 500 & 125 \\
\hline 13 & 500 & 200 & 250 & - & 250 & 250 \\
\hline 15 & 25 & 25 & 25 & 50 & 62.5 & 125 \\
\hline 16 & 50 & 50 & 25 & 25 & 62.5 & 250 \\
\hline 17 & 25 & 50 & 50 & 50 & 125 & 62.5 \\
\hline 18 & 25 & 25 & 50 & 50 & 62.5 & 62.5 \\
\hline 19 & 50 & 25 & 25 & 25 & 125 & 125 \\
\hline $22 \mathbf{a}$ & 25 & 25 & 25 & 25 & 62.5 & 62.5 \\
\hline 22b & 25 & 25 & 50 & 25 & 62.5 & 62.5 \\
\hline $22 c$ & 25 & 50 & 25 & 25 & 62.5 & 62.5 \\
\hline 22d & 50 & 25 & 25 & 50 & 100 & 62.5 \\
\hline $22 \mathrm{e}$ & 25 & 25 & 50 & 50 & 62.5 & 125 \\
\hline 23a & 100 & 50 & 25 & 25 & 100 & 100 \\
\hline 23b & 25 & 25 & 25 & 100 & 62.5 & 62.5 \\
\hline $23 c$ & 25 & 100 & 250 & 25 & 125 & 62.5 \\
\hline 23d & 50 & 25 & 50 & 25 & 250 & 250 \\
\hline $23 e$ & 25 & 100 & 25 & 50 & 62.5 & 125 \\
\hline Amoxicillin & 62.5 & 6.25 & 6.25 & 6.25 & - & - \\
\hline Ketaconazole & - & - & - & - & 31.25 & 31.25 \\
\hline
\end{tabular}

2-yl acetic acid derivatives for pharmacological screening. Various pyrazole derivatives including the drugs butazolidin, celebrex were reported as effective antiinflammatory agents ${ }^{60,61}$. Consequently, it was worthwhile to incorporate pyrazole moieties in the 2 position of phthalazinone nucleus in a hope to yield safe and potent antiinflammatory compounds. Compounds 713, 17-19, 22 b,d and 23 a,d were screened for their antiinflammatory activity.

The screening was conducted in acute inflammatory model. As described by Ramtohul et al. ${ }^{36}$ Carrageenan induced rat paw oedema method was used as follow: Carrageenan (an irritant) at a concentration of $1 \mathrm{mg} \mathrm{mL}^{-1}$ was injected subcutaneously into the hind paw of the rat to produce oedema. Different groups of animals were administered with standard drug indomethacin, the test samples and the vehicle used for the preparation of samples. The increase in the paw volume was measured before and after $3 \mathrm{~h}$ of administration and the results were compared. Ninety healthy albino rats of body weight $170-250 \mathrm{~g}$ were selected and made into nine groups of six animals each. All the animals were kept on fasting for $18 \mathrm{~h}$. One group of animals was kept as control, which received $2 \% \mathrm{w} / \mathrm{v}$ acacia mucilage, which was used to suspend the sample. Another group received the standard drug Indomethacin 1.5 $\mathrm{mg} \mathrm{kg}^{-1}$ body weight intraperitonially. Remaining seven groups of animals received seven different test compounds $(1.5 \mathrm{mg}$ $\mathrm{kg}^{-1}$ body weight) intraperitonially. After $0.5 \mathrm{~h}, 0.1 \mathrm{~mL}$ of w/v carrageenan was injected subcutaneously into the right hind paw of the rats. A mark was made at the right hind paw, which was dipped in the plethismograph up to the mark and the volume was measured immediately and after $3 \mathrm{~h}$. The change in paw volume was compared with that in the vehicle treated control animals. The percentage inhibition of oedema was calculated using the formula:

$$
\% \text { Oedema inhibition }=100-\left(\mathrm{V}_{\text {test }} / \mathrm{V}_{\text {control }}\right) \times 100
$$

The percentage of inhibition was compared with that of the standard drug indomethacin. Interestingly five compounds exhibited good antiinflammatory activity against indomethacin (Table-2).

\begin{tabular}{|c|c|c|c|}
\hline \multicolumn{4}{|c|}{$\begin{array}{c}\text { TABLE-2 } \\
\text { ANTI-INFLAMMATORY ACTIVITY OF } \\
\text { COMPOUNDS 7-13, 17-19, 22b,d AND 23a,d }\end{array}$} \\
\hline Compounds & $\begin{array}{c}\text { Paw oedema } \\
\text { Volume Mean + S.E mL }\end{array}$ & $\begin{array}{c}\text { Percentage } \\
\text { inhibition } \\
\text { of Paw oedema }\end{array}$ & $\begin{array}{c}\text { Dose } \\
\left(\mathrm{mg} \mathrm{kg}^{-1} \text { p.o) }\right.\end{array}$ \\
\hline $\begin{array}{c}2(\%) \\
\text { Gumacacia } \\
\text { (control) }\end{array}$ & $0.62 \pm 0.029$ & & $10 \mathrm{~mL} \mathrm{~kg}^{-1}$ \\
\hline Indomethacine & $0.25 \pm 0.012$ & 59.67 & 1.5 \\
\hline 7 & $0.56 \pm 0.018$ & 30.64 & 50 \\
\hline $8 \mathbf{a}$ & $0.55 \pm 0.013$ & 11.29 & 50 \\
\hline 9 & $0.56 \pm 0.020$ & 9.67 & 50 \\
\hline 10 & $0.52 \pm 1.78$ & 9.67 & 50 \\
\hline 11 & $0.55 \pm 0.015$ & 16.12 & 50 \\
\hline 12 & $0.56 \pm 0.020$ & 9.67 & 50 \\
\hline 13 & $0.55 \pm 0.015$ & 16.12 & 50 \\
\hline 17 & $0.56 \pm 0.018$ & 30.64 & 50 \\
\hline 18 & $0.56 \pm 0.018$ & 30.64 & 50 \\
\hline 19 & $0.46 \pm 0.18$ & 25.8 & 50 \\
\hline $22 b$ & $0.56 \pm 0.018$ & 30.64 & 50 \\
\hline $22 d$ & $0.46 \pm 0.19$ & 25.8 & 50 \\
\hline 23a & $0.56 \pm 0.018$ & 30.64 & 50 \\
\hline 23d & $0.56 \pm 0.019$ & 30.64 & 50 \\
\hline
\end{tabular}

\section{Conclusion}

We reported here the successful synthesis of some novel pyrazolylphthalazine derivatives and acyclo-C-nucleosidesof 6-(2,4,6-trimethylphenyl)-1,2,4-triazolo[3,4-a]phthalazine. Most of the newly synthesized compounds were tested for their antimicrobial and antiinflammatory activity. The antimicrobial activity study showed that all the compounds tested displayed moderate to good antibacterial and antifungal activities against pathogenic strains. In case of antiinflammatory activity, compounds exhibited good activity among the tested compounds.

\section{ACKNOWLEDGEMENTS}

This work was supported by King Saud University, Deanship of Scientific Research, College of Science, Research Center. This work was financially supported by the project "CEITEC-Central European Institute of Technology-Excellent Teams (CZ.1.07/2.3.00/30.0005)" financed from European Social Fund.

\section{REFERENCES}

1. M.S. Chorghade, Drug Discovery and Development; John Wiley \& Sons: Hoboken, New Jersey, Vol. 2 (2007).

2. D. Lednicer and L.A. Mitscher, The Organic Chemistry of Drug Synthesis; John Wiley \& Sons: New York, Chichester, Brisbane, Toronto, Vol. 1 (1977).

3. F. Al-Assar, K.N. Zelenin, E.E. Lesiovskaya, I.P. Bezhan and B.A. Chakchir, Pharm. Chem. J., 36, 598 (2002). 
4. R.W. Carling, K.W. Moore, L.J. Street, D. Wild, C. Isted, P.D. Leeson, S. Thomas, D. O'Connor, R.M. McKernan, K. Quirk, S.M. Cook, J.R. Atack, K.A. Wafford, S.A. Thompson, G.R. Dawson, P. Ferris and J.L. Castro, J. Med. Chem., 47, 1807 (2004).

5. R.P. Jain and J.C. Vederas, Bioorg. Med. Chem. Lett., 14, 3655 (2004).

6. G. Bold, K.H. Altmann, J. Frei, M. Lang, P.W. Manley, P. Traxler, B. Wietfeld, J. Brüggen, E. Buchdunger, R. Cozens, S. Ferrari, P. Furet, F. Hofmann, G. Martiny-Baron, J. Mestan, J. Rösel, M. Sills, D. Stover, F. Acemoglu, E. Boss, R. Emmenegger, L. Lässer, E. Masso, R. Roth, C. Schlachter, W. Vetterli, D. Wyss and J.M. Wood, J. Med. Chem., 43, 2310 (2000).

7. G. Strappaghetti, C. Brodi, G. Giannaccini and L. Betti, Bioorg. Med. Chem. Lett., 16, 2575 (2006).

8. A.D. Lebsack, J. Gunzner, B. Wang, R. Pracitto, H. Schaffhauser, A. Santini, J. Aiyar, R. Bezverkov, B. Munoz, W. Liu and S. Venkatraman, Bioorg. Med. Chem. Lett., 14, 2463 (2004).

9. T. Haack, R. Fattori, M. Napoletano, F. Pellacini, G. Fronza, G. Raffaini and F. Ganazzoli, Bioorg. Med. Chem., 13, 4425 (2005).

10. E.L. Piatnitski, M.A.J. Duncton, A.S. Kiselyov, R. Katoch-Rouse, D. Sherman, D.L. Milligan, C. Balagtas, W.C. Wong, J. Kawakami and J.F. Doody, Bioorg. Med. Chem. Lett., 15, 4696 (2005).

11. K.A. Menear, C. Adcock, F.C. Alonso, K. Blackburn, L. Copsey, J. Drzewiecki, A. Fundo, A. Le Gall, S. Gomez, H. Javaid, C.F. Lence, N.M.B. Martin, C. Mydlowski and G.C.M. Smith, Bioorg. Med. Chem. Lett., 18, 3942 (2008).

12. S. Grasso, G. De Sarro, A. De Sarro, N. Micale, M. Zappalà, G. Puja, M. Baraldi and C. De Micheli, J. Med. Chem., 43, 2851 (2000).

13. Y. Nomoto, H. Obase, H. Takai, M. Teranishi, J. Nakamura and K. Kubo, Chem. Pharm. Bull. (Tokyo), 38, 2179 (1990).

14. M.C. Cardia, S. Distinto, E. Maccioni, L. Bonsignore and A. Delogu, J. Heterocycl. Chem., 40, 1011 (2003).

15. X. Cockcroft, K.J. Dillon, L. Dixon, J. Drzewiecki, F. Kerrigan, V.M. Loh Jr., N.M.B. Martin, K.A. Menear and G.C.M. Smith, Bioorg. Med. Chem. Lett., 16, 1040 (2006).

16. V.M. Loh Jr., X.- Cockcroft, K.J. Dillon, L. Dixon, J. Drzewiecki, P.J. Eversley, S. Gomez, J. Hoare, F. Kerrigan, I.T.W. Matthews, K.A. Menear, N.M.B. Martin, R.F. Newton, J. Paul, G.C.M. Smith, J. Vile and A.J. Whittle, Bioorg. Med. Chem. Lett., 15, 2235 (2005).

17. J. Sung Kim, H.-J. Lee, M.-E. Suh, H.-Y.P. Choo, S.K. Lee, H.J. Park, C. Kim, S.W. Park and C.-O. Lee, Bioorg. Med. Chem., 12, 3683 (2004).

18. A.Z. Haikal, E.S.H. El Ashry and J. Banoub, Carbohydr. Res., 338 2291 (2003).

19. S. Demirayak, A. Karaburun and R. Beis, Eur. J. Med. Chem., 39, 1089 (2004).

20. N. Watanabe, Y. Kabasawa, Y. Takase, M. Matsukura, K. Miyazaki, H. Ishihara, K. Kodama and H. Adachi, J. Med. Chem., 41, 3367 (1998).

21. M. Johnsen, K. Rehse, H. Pertz, J.P. Stasch and E. Bischoff, Arch. Pharmacol., 336, 591 (2003).

22. G.R. Madhavan, R. Chakrabarti, S.K.B. Kumar, P. Misra, R.N.V.S. Mamidi, V. Balraju, K. Kasiram, R.K. Babu, J. Suresh and B.B. Lohray, Eur. J. Med. Chem., 36, 627 (2001).

23. E.M. Lenz, I.D. Wilson, B. Wright, E.A. Partridge, C.T. Rodgers, P.R. Haycock, J.C. Lindon and J.K. Nicholson, J. Pharm. Biomed. Anal., 28, 31 (2002).

24. E.D. Olmo, M.G. Armas, J. Lopez-Perez, G. Ruiz, F. Vargas, A. Gimenez, E. Deharo and A.S. Feliciano, Bioorg. Med. Chem. Lett., 11, 2755 (2001).

25. D.S. Dogruer, E. Kupeli, E. Yesilada and M.F. Sahin, Arch. Pharmcol. 337, 303 (2004)

26. M. Napoletano, G. Norcini, F. Pellacini, F. Marchini, G. Morazzoni, P. Ferlenga and L. Pradella, Bioorg. Med. Chem. Lett., 10, 2235 (2000).

27. A.K. Chakraborti, B. Gopalakrishnan, E. Sobhia and A. Malde, Bioorg. Med. Chem. Lett., 13, 2473 (2003).

28. M. Van der Mey, A. Hatzelmann, I.J. van der Laan, G.J. Sterk, U. Thibaut and H. Timmerman, J. Med. Chem., 44, 2511 (2001).

29. M. Van der Mey, H. Boss, D. Couwenberg, A. Hatzelmann, G.J. Sterk, K. Goubitz, H. Schenk and H. Timmerman, J. Med. Chem., 45, 2526 (2002).
30. K.M. Shubin, V.A. Kuznetsov and V.A. Galishev, Tetrahedron Lett., 45, 1407 (2004).

31. B. Herberich, G.-Q. Cao, P.P. Chakrabarti, J.R. Falsey, L. Pettus, R.M. Rzasa, A.B. Reed, A. Reichelt, K. Sham, M. Thaman, R.P. Wurz, S. Xu, D. Zhang, F. Hsieh, M.R. Lee, R. Syed, V. Li, D. Grosfeld, M.H. Plant, B. Henkle, L. Sherman, S. Middleton, L.M. Wong and A.S. Tasker, J. Med. Chem., 51, 6271 (2008).

32. E. Olmo, B. Barboza, M.I. Ybarra, J.L. López-Pérez, R. Carrón, M.A. Sevilla, C. Boselli and A.S. Feliciano, Bioorg. Med. Chem. Lett., 16, 2786 (2006).

33. J.R. Cashman, T. Voelker, R. Johnson and A. Janowsky, Bioorg. Med. Chem., 17, 337 (2009).

34. T. Sheradsky and R. Moshenberg, J. Org. Chem., 51, 3123 (1986).

35. H.W. Heine, L.M. Baclawski, S.M. Bonser and G.D. Wachob, J. Org. Chem., 41, 3229 (1976).

36. Y.K. Ramtohul, M.N.G. James and J.C. Vederas, J. Org. Chem., 67, 3169 (2002).

37. L.P. Liu, J.M. Lu and M. Shi, Org. Lett., 9, 1303 (2007).

38. A. CsXmpai, K. Körmendy and F. Ruff, Tetrahedron, 47, 4457 (1991).

39. A.S. Amarasekara and S. Chandrasekara, Org. Lett., 4, 773 (2002).

40. J.Y. Hwang, H.-S. Choi and Y.-D. Gong, Tetrahedron Lett., 46, 3107 (2005).

41. R.J. Shuhadolink, C-Nucleosides Antibiotics, Wiley Interscience, New York (1970).

42. E.S. H. El Ashry, N. Rashed and A. Mousaad, J. Carbohydr. Chem., 6, 599 (1987).

43. G.A. El-Hiti, Alex. J. Pharm. Sci., 14, 37 (2000)

44. Y.A. Alsoud and N.A. Almasoudi, Arch. Pharm., 332, 143 (1999).

45. A. Hamed, E.R. Abo-Amaym and E.H. El-Ashry, Nucleosides Nucleotides, 17, 1385 (1998)

46. L.F. Awad and E.S.H.E. Ashry, Carbohydr. Res., 312, 9 (1998).

47. C. Turk, J. Svete, A. Golobic, L. Golic and B. Stanovnik, J. Heterocycl. Chem., 35, 513 (1998).

48. M.A.E. Shaban and A.Z. Nasr, Adv. Heterocycl. Chem., 68, 223 (1997).

49. M.A.E. Shaban and A.E.A. Morgaan, Adv. Heterocycl. Chem., 73, 131 (1999).

50. E.S.H. El-Ashry and Y. El-Kilany, Adv. Heterocycl. Chem., 69, 129 (1997).

51. M.A. El-Hashash, A.Y. Soliman and I.E. El-Shamy, Turk. J. Chem., 36, 347 (2012).

52. C.W. Thornber, J.M. Farrell and D.S. Clarke, Synthesis, 222 (1983).

53. S.M. Fahmy and R.M. Mohareb, Liebigs Ann. Chem., 1492 (1985).

54. M. Ishii, M. Iwahana, I. Mitsui, M. Minami, S. Imagawa, A. Tohgo and A. Ejima, Anticancer Drugs, 11, 353 (2000).

55. R. Noto, F. Buccheri, G. Cusmano, M. Gruttadauria and G. Werber, J. Heterocycl. Chem., 28, 1421 (1991).

56. B.D. Robert and W. Robin, Eur. Patent, 2,895 (1979); Chem. Abstr., 92, 76533v (1980).

57. M. Van der Mey, A. Hatzelmann, G.P.M. Van Klink, I.J. Van der Laan, G.J. Sterk, U. Thibaut, W.R. Ulrich and H. Timmerman, J. Med. Chem., 44, 2523 (2001).

58. W.O. Foye, Principle of Medicinal Chemistry, Philadeliphia, London, edn. 3, p. 516 (1989).

59. W.O. Foye, T.L. Lemke and D.A. Williams, Principle of Medicinal Chemistry, A Waverly Company, edn. 4, p. 554 (1989).

60. T.D. Penning, J.J. Talley, S.R. Bertenshaw, J.S. Carter, P.W. Collins, S. Docter, M.J. Graneto, L.F. Lee, J.W. Malecha, J.M. Miyashiro, R.S. Rogers, D.J. Rogier, S.S. Yu, G.D. Anderson, E.G. Burton, J.N. Cogburn, S.A. Gregory, C.M. Koboldt, W.E. Perkins, K. Seibert, A.W. Veenhuizen, Y.Y. Zhang and P.C. Isakson, J. Med. Chem., 40, 1347 (1997).

61. C.A. Winter, E.A. Risley and G.W. Nuss, Proc. Soc. Exp. Biol. Med., 111, 544 (1962).

62. M.A. El-Hashash, A.Y. El-Kady, M.A. Taha and I.E. El-Shamy, Chinese J. Chem., 30, 616 (2012).

63. I.E. El-Shamy, H.M. Bakeer, K.M. Al-Shamrani, A.M. Abdel-Mohsen and M.M. Al-Shehri, Life Sci. J., 11, 94 (2014). 